\title{
Analytic theory of Titan's Schumann resonance: Constraints on ionospheric conductivity and buried water ocean
}

\author{
Christian Béghin ${ }^{\mathrm{a}, *}$, Orélien Randriamboarison ${ }^{\mathrm{a}}$, Michel Hamelin ${ }^{\mathrm{b}}$, Erich Karkoschka ${ }^{\mathrm{c}}$, Christophe Sotin ${ }^{\mathrm{d}}$, \\ Robert C. Whitten ${ }^{\mathrm{e}}$, Jean-Jacques Berthelier ${ }^{\mathrm{b}}$, Réjean Grard ${ }^{\mathrm{f}}$, Fernando Simões ${ }^{\mathrm{g}}$
}

${ }^{a}$ LPC2E-CNRS-Université d'Orléans, 3A, Ave. Recherche Scientifique, 45071 Orléans Cedex 2, France

${ }^{\mathrm{b}}$ LATMOS-IPSL, Université Pierre et Marie Curie, 4, Place Jussieu, 75252 Paris Cedex 05, France

${ }^{\mathrm{C}}$ Lunar and Planetary Laboratory, University of Arizona, Tucson, AZ 85721-0092, USA

d Jet Propulsion Laboratory, California Institute of Technology, Pasadena, CA 91109, USA

e SETI Institute, 189 Bernardo Ave., Mountain View, CA 94043, USA

${ }^{\mathrm{f}}$ RSSD, ESA-ESTEC, European Space Agency, Keplerlaan 1, 2200 AG Noordwijk, The Netherlands

${ }^{\mathrm{g}}$ NASA-GSFC, Heliophysics Science Division, Greenbelt, MD 20771, USA

\section{A R T I C L E I N F O}

\section{Article history:}

Received 15 June 2011

Revised 31 January 2012

Accepted 5 February 2012

Available online 15 February 2012

\section{Keywords:}

Interiors

Lightning

Saturn, Magnetosphere

Titan, Atmosphere

Titan, Interior

\begin{abstract}
A B S T R A C T
This study presents an approximate model for the atypical Schumann resonance in Titan's atmosphere that accounts for the observations of electromagnetic waves and the measurements of atmospheric conductivity performed with the Huygens Atmospheric Structure and Permittivity, Wave and Altimetry (HASI-PWA) instrumentation during the descent of the Huygens Probe through Titan's atmosphere in January 2005. After many years of thorough analyses of the collected data, several arguments enable us to claim that the Extremely Low Frequency (ELF) wave observed at around $36 \mathrm{~Hz}$ displays all the characteristics of the second harmonic of a Schumann resonance. On Earth, this phenomenon is well known to be triggered by lightning activity. Given the lack of evidence of any thunderstorm activity on Titan, we proposed in early works a model based on an alternative powering mechanism involving the electric current sheets induced in Titan's ionosphere by the Saturn's magnetospheric plasma flow. The present study is a further step in improving the initial model and corroborating our preliminary assessments. We first develop an analytic theory of the guided modes that appear to be the most suitable for sustaining Schumann resonances in Titan's atmosphere. We then introduce the characteristics of the Huygens electric field measurements in the equations, in order to constrain the physical parameters of the resonating cavity. The latter is assumed to be made of different structures distributed between an upper boundary, presumably made of a succession of thin ionized layers of stratospheric aerosols spread up to $150 \mathrm{~km}$ and a lower quasi-perfect conductive surface hidden beneath the non-conductive ground. The inner reflecting boundary is proposed to be a buried water-ammonia ocean lying at a likely depth of 55-80 km below a dielectric icy crust. Such estimate is found to comply with models suggesting that the internal heat could be transferred upwards by thermal conduction of the crust, while convective processes cannot be ruled out.
\end{abstract}

(c) 2012 Elsevier Inc. All rights reserved.

\section{Introduction}

Titan, Saturn's largest satellite, is the only moon in the Solar System with a dense atmosphere and stable liquid hydrocarbon lakes on its surface. Its global properties (mass, radius, density) are similar to those of its cousin icy jovian satellites Ganymede

\footnotetext{
* Corresponding author. Fax: +33 (0) 238633412 .

E-mail addresses: cbeghin@cnrs-orleans.fr (C. Béghin), randriam@cnrs-orleans.fr (O. Randriamboarison), michel.hamelin@latmos.ipsl.fr (M. Hamelin), erich@pirl. lpl.Arizona.EDU (E. Karkoschka), csotin@jpl.nasa.gov (C. Sotin), rwhitten@pacbell. net (R.C. Whitten), jean-jacques.berthelier@latmos.ipsl.fr (J.-J. Berthelier), rgrard@rssd.esa.int (R. Grard), fernando.a.simoes@nasa.gov (F. Simões).
}

and Callisto. The discovery by the Galileo mission of their own magnetic fields, partly intrinsically produced (Ganymede) and partially induced by the variations of the jovian magnetic field, has led to the interpretation that internal water oceans are present beneath their icy crust (Schubert et al., 2004). Discovering a buried ocean at Titan would mean that this moon is a likely world of organic molecules and liquid water, two of the necessary ingredients for life to form and develop. Unfortunately, the magnetic measurements from the Cassini orbiter (Dougherty et al., 2004) do not reach the sensitivity required for a similar detection at Titan. The reasons for that are manifold, namely: (i) a fainter signal, since Saturn's magnetic field is weaker at Titan's orbit than that of Jupiter at its moons, (ii) a larger safe flyby distance of the 
spacecraft above the surface, and more specifically, (iii) the shielding effect due to Titan's ionosphere.

Thanks to Titan's dense atmosphere, the presence of a buried ocean might be detected by measuring the non-synchronous rotation of the crust (i.e., the length of day) due to the periodical exchange of angular momentum between the atmosphere and the crust (Tokano and Neubauer, 2005). An initial analysis of the location of geological features at different times led the Cassini Radar team (Stiles et al., 2008; Lorenz et al., 2008) to propose a non-synchronous rotation rate of $1.1 \times 10^{-3}$ degree/day that would have been consistent with this model. However, the discovery of an error in the data reduction led to reconsider the rate as being rather $3 \times 10^{-4}$ degree/day (Stiles et al., 2010). Since this value is strongly correlated with the precession, it lies within the error bar of a non-synchronous rotation. Furthermore, a more elaborate model of angular momentum exchange (Tokano et al., 2011) predicts much smaller displacement, with amplitudes of polar motion from 100 to $1000 \mathrm{~m}$ for crustal thickness of $70-20 \mathrm{~km}$, respectively. Given that the spatial resolution of the radar is several times larger than the $300 \mathrm{~m}$ surface sampling, it appears that the Cassini Radar observations could detect the non-synchronous component related to the presence of a buried ocean if the thickness of the crust is less than $20 \mathrm{~km}$ (Ö. Karatekin, Royal Observatory of Belgium, private communication).

Independently, the possibility to diagnose the shallow interior of planets, using the properties of Extremely Low Frequency (ELF) waves trapped within their atmospheric cavity, was suggested by Sentman (1990a) as a way to reveal the liquid metallic hydrogen surface of Jupiter. To date, the only observation that seems consistent with the presence of a subsurface water ocean at Titan is that of the ELF signal collected by the Huygens Probe (Béghin et al., 2010), as it descended through the atmosphere on January 14, 2005.

The Permittivity, Wave and Altimetry (PWA) experiment (Grard et al., 1995), a subsystem of the Huygens Atmospheric Structure Instrument (HASI) (Fulchignoni et al., 2002), was designed to perform measurements of electron and ion conductivities, ELF and VLF (Very Low Frequency) waves during the descent through Titan's atmosphere (Lebreton et al., 2005). One of the most exciting but speculative goals of the PWA experiment dealt with the possible existence of lightning activity in Titan's atmosphere and the associated Schumann resonance (Schumann, 1952; Sentman, 1995), hereinafter referred to as "SR". Subsequently, the presence of a ground or subterranean conductive surface, such a liquid water ocean predicted by theoretical models (e.g., Grasset and Sotin, 1996), was expected to be a major scientific return of the mission. Preliminary data analyses revealed indeed that the $36 \mathrm{~Hz}$ signal observed with the PWA instrument (Grard et al., 2006) might satisfactorily fit the range of values predicted by models to be the second eigenmode of the SR (Nickolaenko et al., 2003; Simões et al., 2007). Given the absence of any acknowledged lightning activity on Titan after 72 flybys from 2005 to 2010 (Fischer and Gurnett, 2011), it was proposed that the ELF turbulence generated within the ionospheric currents driven by the co-rotating plasma flow of Saturn's magnetosphere (e.g., Sittler et al., 2009) might be the power source able to excite a "Schumann-like" resonance (Béghin et al., 2007). After reanalyzing the characteristics of the $36 \mathrm{~Hz}$ signal in term of height profile and spin modulation, including a few attempts at identifying possible source regions from the measurements performed by the plasma and wave instruments on board the Cassini orbiter, the proposed powering mechanism was further developed in follow-up articles (Béghin et al., 2009, 2010).

In the latter papers, because of the absence of any detectable radial electric field component of the $36 \mathrm{~Hz}$ signal, we were led to consider a mode of propagation with horizontal polarization. This assumption must be revisited and we consider instead the properties of waves with mixed horizontal and radial polarizations, called Transverse Electric and Magnetic (TEM) modes, known to be those of Earth's SR (Wait, 1962). However, in a stratified cavity, bounded by an icy crust instead of a conductive ground and a multi-layered profile of atmospheric conductivity, the TEM waves are known to degenerate into Longitudinal Section Magnetic (LSM) modes (Collin, 1991), whose properties are shown below to be notably different from those of Earth's SR.

We first establish the basic equations relevant to Titan conditions in Sections 2 and 3, referring to previous works and to Appendix A for some mathematical developments. After deriving the expressions of field components in Section 3 and the modal equations in Section 4, the formalism is subsequently applied to the PWA observations in Section 5 in order to constrain the parameters of the cavity. Making use of the Huygens Probe dynamics data deduced from the Descent Imager Spectral Radiometer (DISR) observations (Karkoschka et al., 2007), we retrieve in Section 5.4 a few case-studies of electric field strength profiles which compare favorably with Huygens measurements throughout the descent.

Section 6 contains discussions regarding the two main results of our study, the first one being the constrained upper atmosphere electron conductivity profile above the Galactic Cosmic Ray (GCR) layer at around $60 \mathrm{~km}$ revealed by the PWA-MIP (Mutual Impedance Probe) and PWA-RP (Relaxation Probe) instruments (Hamelin et al., 2007; López-Moreno et al., 2008). We invoke a probable relationship with thin aerosol layers and we consider a possible correlation with the zonal wind profile measured by the Doppler Wind Experiment (DWE) during the descent (Bird et al., 2005). The second major result concerns the crust characteristics and the presence of a buried conductive ocean, whose constrained parameters are discussed in terms of Titan's interior thermal dynamics. Although only few ground truth and remote sensing measurements support the relevance of the constraints derived from our work, we are able to conclude in Section 7 that our interpretation of PWA-ELF data is consistent with recent models of Titan's interior. The cavity appears to be bounded upwards by thin ionized layers of aerosols, at least up to an altitude of $140 \mathrm{~km}$ (López-Moreno et al., 2008) and downwards by a water-ammonia ocean buried beneath a semi-rigid icy crust, most likely $55-80 \mathrm{~km}$ thick.

We revisit, in Appendix B, a few experimental open points that have been subject of recurring questions regarding the validity of the PWA measurements, such as: (i) the absence of multi-SR modes that should have been observed in addition to the $36 \mathrm{~Hz}$ line, (ii) the disappearance of the signal $16 \mathrm{~s}$ after landing, and (iii) a presumably artifact induced by boom-antenna vibrations at $36 \mathrm{~Hz}$ possibly triggered by wind-blasts or by the descent velocity airflow (Béghin et al., 2007).

\section{Formulation of the problem}

Once compendious analyses of the raw data had failed to reveal any convincing signature of a radial electric field component (Béghin et al., 2009), it was wrongly concluded that the polarization might be essentially horizontal. Such an erroneous conclusion came from the inaccuracy in the time resolution of the $36 \mathrm{~Hz}$ signal strength which did not exhibit convincing correlation with the fast variations of the tilt of Huygens vertical axis (Section 6.1), as could be expected from the presence of a significant radial electric field. When the excitation comes from a horizontal electric dipole, or the system of azimuthal current sheets proposed by Béghin et al. (2007), horizontal and radial polarized waves coexist (Bahar and Fitzwater, 1983), and trapped TEM modes have both radial and horizontal electric components, but no radial magnetic field component (Galejs, 1972). However, as the structure of Titan's cavity 
is stratified, we must instead consider the LSM modes which are known to exhibit high quality factors in wave-guides filled with dielectric layers (Collin, 1991).

Though the following analytic treatment presents physical analogies with that of TEM modes of Earth's SR (Greifinger and Greifinger, 1978; Sentman, 1990b; Füllekrug, 2000; Nickolaenko et al., 2003), both approaches differ significantly, not only because ionospheric horizontal current sources are involved instead of vertical currents, as would be the case for ground-to-cloud return-strokes, but also because of the presence of stratifications which requires appropriate treatments at the layer interfaces (Wait, 1962; Collin, 1991). The physical concepts are nevertheless of the same nature, in the sense that we treat the scattering mechanism of an incident plane wave over a perfectly conductive inner surface, which is found to be a satisfying approximation compared to results of full-wave expansion treatments (Bahar and Fitzwater, 1983). The conductivity of Titan's icy-crust $\left(\sim 0.2 \mathrm{nS} \mathrm{m}^{-1}\right)$ measured by the PWA-MI instrument (Grard et al., 2006) is too low to reflect ELF waves, and its permittivity $(\sim 2.5)$ is found consistent with the value derived from the Cassini Radar Scatterometer (Wye et al., 2007). Therefore, we consider a subterranean conductive surface, presumed to be a water-ammonia liquid ocean buried under an icy crust made of dielectric material (Fig. 1). For the electrical properties of the atmosphere, we consider the electron conductivity profile deduced from the PWA-MIP-RP instruments up to about $90 \mathrm{~km}$, including the GCR layer. At higher altitudes, the performance of PWA-MIP instrument is doubtful (Hamelin et al., 2007; Béghin et al., 2009), due to a suspected non-nominal configuration involving probably the boom-antenna release system prior to the main parachute jettison (Lebreton et al., 2005). Moreover, above the GCR layer, the PWA-RP measurements exhibited discontinuities attributed to stratifications of few hundred-meter size with the occurrence of "plateaus", i.e., time intervals during which the response of the probe potential remains constant for 6-32 s, at about 100 and $72 \mathrm{~km}$ respectively (López-Moreno et al., 2008). Thus, in the absence of high altitude measurements applicable to a global planetary scale, we will resort to case-study models with one-scale-height atmospheric electron conductivity yielding a best fit between retrieved and experimental field profiles (Sections 4 and 5.5).

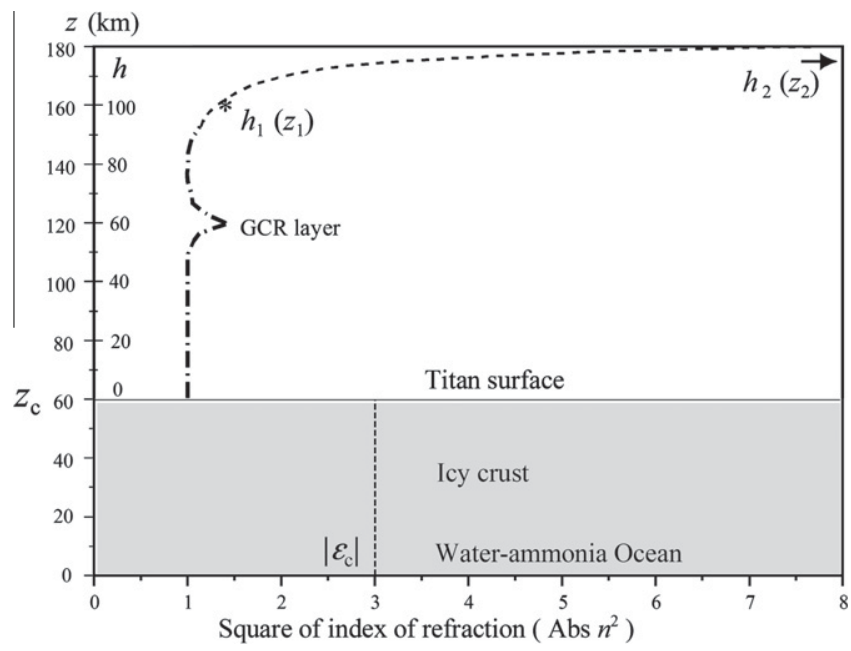

Fig. 1. Model of Titan's layered cavity. Dash-dot line: altitude profile of $\left|n^{2}\right|$ measured by the PWA-MIP-RP instruments from 0 to $90 \mathrm{~km}$ altitude, and analytic continuation upwards (dot line). Vertical scales: $z$ is referring to inner boundary (ocean), $h$ is the altitude above Titan surface; $h_{1}\left(z_{1}\right)$ and $h_{2}\left(z_{2}\right)$ are the conduction and diffusion altitudes, respectively (see text in Section 4). Values of each parameter are given in Table 2 for six case-studies.
In a spherical geometry the radial structure is assumed to be arranged in series of discrete shell slabs wherein the electrical properties are homogeneous, so that we may apply the following usual Maxwell equations

$\nabla \times \mathbf{H}=\mathbf{J}_{e}+\frac{\partial \mathbf{D}}{\partial t} ; \nabla \cdot \mathbf{B}=0 ; \nabla \times \mathbf{E}=-\frac{\partial \mathbf{B}}{\partial t} ; \mathbf{D}=\varepsilon_{\mathbf{0}} n^{2} \mathbf{E} ; \mathbf{B}=\mu_{0} \mathbf{H}$,

where $\mathbf{H}$ and $\mathbf{E}$ are the magnetic and electric vectors, $\mathbf{D}$ and $\mathbf{B}$ the electric displacement and the magnetic induction; $\varepsilon_{0}$ and $\mu_{0}$ are the free space permittivity and permeability, and $\mathbf{J}_{e}$ is a possible induced surface-current distribution, as for instance, at the inner perfectly conductive boundary. The complex relative permittivity $\varepsilon$ of the medium is written in terms of conductivity as

$\varepsilon \equiv n^{2}=\operatorname{Re} \varepsilon+i \operatorname{Im} \varepsilon, \quad$ with $\operatorname{Im} \varepsilon=\frac{\sigma}{\omega \varepsilon_{0}}$,

where $n$ is the index of refraction and $\sigma$ the scalar conductivity. All quantities are assumed to vary versus time as $\exp (-i \omega t)$, where $f$ is the wave frequency, $\omega=2 \pi f$ and $i=\sqrt{ }-1$. Introducing the RiemannLorenz vector potential $\mathbf{A}$, from the second and third equations in Eq. (1) one readily obtains

$\mathbf{B}=\nabla \times \mathbf{A}$ and $\nabla \times\left(\mathbf{E}+\frac{\partial \mathbf{A}}{\partial t}\right)=0$.

Introducing the scalar potential function $\Psi$, the second equation in Eq. (3) yields

$\nabla \Psi+\mathbf{E}+\frac{\partial \mathbf{A}}{\partial t}=0$

Applying the curl operator to the first equation in Eq. (3), and introducing the identity $k^{2} \equiv \omega^{2} \mu_{0} \varepsilon_{0}$, where $k$ is the free space wave number defined as $2 \pi / \lambda$, and $\lambda$ is the free space wavelength, we get the wave equation as a function of both potentials

$\nabla \times \nabla \times \mathbf{A}=i \frac{k^{2} n^{2}}{\omega} \nabla \Psi+k^{2} n^{2} \mathbf{A}$.

Using a procedure similar to that applied for the computation of terrestrial SR parameters (Sentman, 1990b), the wave Eq. (5) may be reduced into a set of two independent differential equations for each potential $\mathbf{A}$ and $\Psi$. The procedure, developed in Appendix A, is basically the same as for radially polarized TM modes, i.e., when the radial magnetic field is forced to zero, with the consequence for the vector potential to have only a radial component $A_{r}$. The resulting Eqs. (A8a) and (A8b) are known as particular forms of generalized Helmholtz wave equations applied to inhomogeneous cavities, and will be referred to in the next sections whenever needed.

\section{Field expressions and approximate solution of wave equation}

First, we define the initial boundary conditions for the horizontal components of electric and magnetic fields obeying Eqs. (3) and (4), i.e.,

$\left\{\mathbf{E}_{\theta}=\mathbf{E}_{\varphi}=0\right\}_{z=0} \Rightarrow\left\{\hat{\boldsymbol{\theta}} \cdot \nabla \Psi_{r}=0 ; \hat{\boldsymbol{\varphi}} \cdot \nabla \Psi_{r}=0 ; \hat{\mathbf{r}} \cdot \nabla \Psi_{r} \neq 0 ; \Psi_{r}=0\right\}_{z=0}$

$\left\{\mathbf{H}_{\theta} \neq 0 ; \mathbf{H}_{\varphi} \neq 0\right\}_{z=0} \Rightarrow\left\{A_{r}=A_{0}\right\}_{z=0}$,

where $A_{0}$ is the amplitude of the vector potential at the lower conductive boundary $(z=0)$ after performing the change of variable $z=r-a+z_{c}$, where $a$ is the mean Titan's radius and $z_{c}$ the thickness of the crust (Fig. 1). Starting from the wave equations Eqs. (A8a) and (A8b), we may proceed either with the vector potential or the scalar potential, independently of each other. The field expressions 
deduced from Eqs. (3), (4), (A3), and (A7), are written in terms of the vector potential as

$\mathbf{H}_{\theta}=\hat{\boldsymbol{\theta}} \frac{A_{r}}{a \mu_{0} \sin \theta} \frac{\partial Y(\theta, \varphi)}{\partial \varphi} ; \mathbf{H}_{\varphi}=-\hat{\boldsymbol{\varphi}} \frac{A_{r}}{a \mu_{0}} \frac{\partial Y(\theta, \varphi)}{\partial \theta}$,

$\mathbf{E}_{\mathbf{r}}=\hat{\mathbf{r}} \frac{i \omega l(l+1)}{k^{2} a^{2}}\left(\frac{A_{r}}{n^{2}}\right) Y(\theta, \varphi) ; \mathbf{E}_{\theta}=\hat{\boldsymbol{\theta}} \frac{-i \omega}{k^{2} a}\left(\frac{1}{n^{2}} \frac{\partial A_{r}}{\partial z}\right) \frac{\partial Y}{\partial \theta} ;$

$\mathbf{E}_{\varphi}=\hat{\boldsymbol{\varphi}} \frac{i \omega}{k^{2} a \sin \theta}\left(\frac{1}{n^{2}} \frac{\partial A_{r}}{\partial z}\right) \frac{\partial Y}{\partial \varphi}$

Note that the LSM modes are entirely defined by a single scalar function $A_{r}$, namely the Hertzian potential (Collin, 1991). Similarly, the fields can be written in terms of the scalar potential

$$
\begin{aligned}
& \mathbf{H}_{\theta}=\hat{\boldsymbol{\theta}} \frac{-i k^{2} n^{2} a}{\left[k^{2} n^{2} a^{2}-l(l+1)\right] \mu_{0} \sin \theta} \frac{\partial \Psi_{r}}{\partial z} \frac{\partial Y}{\partial \varphi} \\
& \mathbf{H}_{\varphi}=\hat{\boldsymbol{\varphi}} \frac{i k^{2} n^{2} a}{\left[k^{2} n^{2} a^{2}-l(l+1)\right] \mu_{0}} \frac{\partial \Psi_{r}}{\partial z} \frac{\partial Y}{\partial \theta}, \\
& \mathbf{E}_{r}=\hat{\mathbf{r}} \frac{l(l+1)}{k^{2} n^{2} a^{2}-l(l+1)} \frac{\partial \Psi_{r}}{\partial z} Y(\theta, \varphi) ; \mathbf{E}_{\theta}=\hat{\boldsymbol{\theta}} \frac{-\Psi_{r}}{a} \frac{\partial Y}{\partial \theta} \\
& \mathbf{E}_{\varphi}=\hat{\boldsymbol{\varphi}} \frac{-\Psi_{r}}{a \sin \theta} \frac{\partial Y}{\partial \varphi}
\end{aligned}
$$

We may derive an approximate solution of Eq. (A8b) for the Hertzian potential, starting from the lower boundary $z=0$ up to the ionospheric boundary at the altitude $h_{1}$ (Fig. 1). The latter is defined as the conduction boundary (Greifinger and Greifinger, 1978), where the conduction current becomes equal to the displacement current. According to Eq. (2), this condition reads

$n_{h_{1}}^{2}=1+i \frac{\sigma}{\omega \varepsilon_{0}} \equiv 1+i$.

In the atmosphere up to $h_{1}$, the electron conductivity measured by PWA-MIP-RP instruments is sufficiently low to consider $\left|n^{2}\right|$ close to 1. Applying the wave equation within slabs, where $n^{2}$ is assumed to be constant, Eq. (A8b) simplifies as

$\frac{\partial^{2} A_{r}}{\partial z^{2}}+K_{j}^{2} A_{r}=0 \quad$ with $K_{j}^{2}=k^{2}\left(n_{j}^{2}-\gamma_{l}\right) \quad$ and $\gamma_{l}=\frac{l(l+1)}{k^{2} a^{2}}$,

where $j$ is the slab index, starting from $j=1$ at the bottom of the icy crust ( $z=0), K_{j}$ is the radial wave number, $l$ is the SR eigenmode degree. As long as we consider ELF waves with free space wavelengths $\lambda$ of the order or even larger than $a$, the value of $K_{j}^{2}$ in the Eq. (10) is of the order of magnitude of $(2 \pi / \lambda)^{2}$, thus $\left|K_{j}^{2} z^{2}\right| \ll 1$ everywhere in the cavity for $K_{j}^{2}$ positive, negative or complex. Although the general solution of Eq. (10) is known to be a combination of Bessel functions for the complex variable $K_{j}$, we consider here the exponential thinshell approximation (Galejs, 1972), whose solution is a combination of upgoing and downgoing harmonic waves, as

$A_{r}(z)=\alpha \exp \left(i K_{j} z\right)+\beta \exp \left(-i K_{j} z\right)$,

where $\alpha$ and $\beta$ are determined by the initial boundary conditions Eqs. (6a) and (6b), i.e., $\alpha=\beta=A_{0} / 2$. Thus, the solution of Eq. (11) within the crust yields

$A_{r}(z)=A_{0} \cos \left(K_{1} z\right) ; \frac{1}{\varepsilon_{c}} \frac{\partial A_{r}}{\partial z}=-A_{0} \frac{K_{1}}{\varepsilon_{c}} \sin \left(K_{1} z\right)$ for $z<z_{c}$,

where $K_{1}$ is given by Eq. (10) with $n^{2}=\varepsilon_{\mathrm{c}}$. We apply now the interface conditions to each crossing of successive slabs, in such a way that the radial component of the displacement vector $\boldsymbol{D}$ and both horizontal electric and magnetic field components are continuous. Starting from the ground surface, this reads
$\left(A_{r} Y\right)_{Z_{\bar{C}}^{-}}=\left(A_{r} Y\right)_{Z_{C}^{+}} ;\left(\frac{1}{\varepsilon_{c}} \frac{\partial A_{r}}{\partial z}\right)_{Z_{\bar{C}}^{-}}=\left(\frac{1}{n_{2}^{2}} \frac{\partial A_{r}}{\partial z}\right)_{Z_{C}^{+}}$,

where $z_{c}^{-}$and $z_{c}^{+}$refer to the levels below and above the crust surface, respectively. Applying to Eq. (11) the continuity conditions for $A_{r}$ and its first derivative, we get the wave expression that is valid upwards throughout the atmosphere as long as each slab is supposed to be homogeneous

$$
\begin{aligned}
\left(A_{r}\right)_{Z>Z_{c}}=A_{0}[ & \cos \left(K_{1} z_{c}\right) \cos K_{2}\left(z-z_{c}\right) \\
- & \left.\frac{n_{2}^{2} K_{1}}{\varepsilon_{c} K_{2}} \sin \left(K_{1} z_{c}\right) \sin K_{2}\left(z-z_{c}\right)\right], \\
\frac{1}{n_{2}^{2}}\left(\frac{\partial A_{r}}{\partial z}\right)_{z>z_{c}}= & -A_{0}\left[\frac{K_{2}}{n_{2}^{2}} \cos \left(K_{1} z_{c}\right) \sin K_{2}\left(Z-Z_{c}\right)\right. \\
& \left.+\frac{K_{1}}{\varepsilon_{c}} \sin \left(K_{1} z_{c}\right) \cos K_{2}\left(Z-Z_{c}\right)\right],
\end{aligned}
$$

where $K_{2}$ is the complex radial wave number solution of Eq. (10) in the atmosphere when $n_{2}^{2} \approx 1$. For $l=2, f=36 \mathrm{~Hz}$ and $a \sim 2600 \mathrm{~km}$ it can be seen from Eq. (10) that $\gamma_{2} \sim 1.56$; thus $K_{2}^{2}$ is almost purely real negative as long as $n_{2}^{2} \sim 1$, so that $K_{2}$ is lying nearly on positive imaginary axis. This fact does not imply that the wave is damped, because the phase increment $\left|K_{2} z\right|$ through the atmospheric portion of the cavity is nearly zero. Thus, the first order expansions of Eqs. (14) and (15) are real, so that $A_{r}$ does not depart significantly from its initial value $A_{0}$ at least up to the ionospheric boundary $h_{1}$, although its first derivative $\partial A_{r} / \partial z$ is not zero. As a consequence, the horizontal magnetic field components keep nearly constant amplitude versus $z$ for given $\theta$ and $\varphi$ by virtue of Eq. (7a). Introducing Eq. (15) in the last two expressions of Eq. (7b) one can check that the horizontal electric components increase linearly from 0 at $z=0$, up to their maximum value reached in the vicinity of $h_{1}$, while apart from the GCR layer, the radial electric component is almost constant from the ground up to around $h_{1}$ (Section 5.3).

\section{Modal equation}

The modal expression of the eigenmodes of Titan's cavity is derived using the approach outlined by Greifinger and Greifinger (1978) for the terrestrial SR in cylindrical geometry and later on in spherical geometry by Sentman (1990b) and for Titan by Nickolaenko et al. (2003). Here, the only significant difference with respect to the Earth conditions is the presence of the subsurface icy-crust, whose physical parameters must be included in the model. We saw that the amplitude of $A_{r}$, given by Eq. (14), is almost constant as long as $\left|K_{2} z\right| \ll 1$. Thus, we may perform the integration of the height-gain Eq. (A7) for $\Psi_{r}$ throughout the cavity up to some distance above $h_{1}$, where $A_{r}$ may be taken outside the integral. Inside the icy crust, the result is straightforward

$\Psi_{r}(z)=i \omega A_{0}\left(1-\gamma_{l} / \varepsilon_{c}\right) z$.

Boundary conditions similar to Eq. (13) applied to the scalar potential read

$$
\begin{aligned}
\Psi_{r, j} & =\Psi_{r, j-1}+\left(z-z_{j-1}\right)\left(\frac{\partial \Psi_{r}}{\partial z}\right)_{j} \text { and }\left(\frac{n_{j}^{2}}{K_{j}^{2}} \frac{\partial \Psi_{r}}{\partial z}\right)_{j} \\
& =\left(\frac{n_{j-1}^{2}}{K_{j-1}^{2}} \frac{\partial \Psi_{r}}{\partial z}\right)_{j-1} \quad \text { for } z_{j-1} \leqslant z<z_{j},
\end{aligned}
$$

where $j$ and $j-1$ are the indices respectively above and below any slab boundary. It can be checked from Eq. (17) that $\Psi_{r}$ is continuous across the ground surface. This is not the case for its derivative which exhibits an abrupt $90^{\circ}$ phase shift while the permittivity 
drops from $\varepsilon_{c}>1$ down to 1 . However, according to Eqs. (8b) and (17), the horizontal electric components grow linearly in the atmosphere, as we found above using the vector potential (Section 3). For simplification purposes, we choose the analytical approximation of one-scale-height exponential profile which is shown to predict satisfactorily the Earth's SR modes for normal ionospheric conditions (Füllekrug, 2000). The presence of small irregularities, such as the GCR layer (Fig. 1), could introduce minor deviations which are found to have a weak effect on field profiles (Section 5.3). The one-scale-height exponential profile referring to the altitude of conduction boundary $h_{1}$, where $n^{2}=1+i$, reads

$n^{2}=1+i \exp \left(\frac{z-z_{1}}{\zeta}\right)$

where $\zeta$ is the scale height and $z_{1}=z_{\mathrm{c}}+h_{1}$ (Fig. 1). Using Eq. (18), and integrating Eq. (A7) from the ground up to $z$ above $z_{1}$, yields

$$
\begin{aligned}
\Psi_{r}(z) & =\Psi_{r}\left(z_{c}\right)+i \omega A_{0} \int_{z_{c}}^{z}\left(1-\frac{\gamma_{l}}{n^{2}}\right) d z \\
& =\Psi_{r}\left(z_{c}\right)+i \omega A_{0}\left[z_{c}\left(\gamma_{l}-1\right)+z-\gamma_{l}\left(z_{1}-i \frac{\pi}{2} \zeta\right)\right] .
\end{aligned}
$$

Substituting in Eq. (19) the value of $\Psi_{r}\left(z_{c}\right)$ given by Eq. (16), we obtain

$\Psi_{r}(z)=i \omega A_{0}\left[z-\gamma_{l}\left(z_{1}-z_{c}+z_{c} / \varepsilon_{c}-i \frac{\pi}{2} \zeta\right)\right] ;\left(z>z_{1}+\zeta\right)$.

In regions well above $z_{1}, n^{2}$ reaches high values such as the last term with gradient of $n^{-2}$ in Eq. (A8a) vanishes. Similarly, we may ignore the term $\gamma_{l}$ compared to $n^{2}$ in the coefficient of $\Psi_{r}$. Then, using Eq. (9) and the identity $k^{2} \equiv \mu_{0} \varepsilon_{0} \omega^{2}$, the wave equation reduces to

$\frac{\partial^{2} \Psi_{r}}{\partial z^{2}}+i \mu_{0} \omega \sigma(h) \Psi_{r}=0 ; \quad\left(z \gg z_{1}+\zeta\right)$.

We define the upper ionospheric boundary as $z_{2}=h_{2}+z_{c}$, where $h_{2}$ (Fig. 1) is the altitude of diffusion for the magnetic components and the ultimate reflection boundary for the horizontal electric components (Greifinger and Greifinger, 1978). Extrapolation of the conductivity profile given by Eq. (18), yields the expression of the conductivity at $z=z_{2}$

$\sigma(z)=\sigma_{2}\left(z_{2}\right) \exp \left(\frac{z-z_{2}}{\zeta}\right)$ with $\sigma_{2}\left(z_{2}\right)=\varepsilon_{0} \omega \exp \left(\frac{z_{2}-z_{1}}{\zeta}\right)$.

Performing the change of variable $v=\exp \left[i \pi / 4+\left(z-z_{2}\right) / 2 \zeta\right]$, Eq. (21) becomes

$\frac{\partial^{2} \Psi_{r}}{\partial^{2} v}+\frac{1}{v} \frac{\partial \Psi_{r}}{\partial v}+4 \mu_{0} \omega \sigma_{2} \zeta^{2} \Psi_{r}=0$.

Giving the conductivity $\sigma_{2}$ a value such that $4 \mu_{0} \omega \sigma_{2} \zeta^{2}=1$ at the boundary $h_{2}$, Eq. (23) takes the form of a Bessel's differential equation whose solution is proportional to the zeroth order Hankel's function of first kind $H_{0}^{(1)}(v)$ (Abramowitz and Stegun, 1972). Above $z_{2}$ the function tends rapidly towards zero when $v \rightarrow \infty \exp i \pi / 4$ for $z \gg z_{2}$, whereas below $z_{2}$, where $v \rightarrow 0$ exp $i \pi / 4$ for upgoing waves, the small argument expansion of Hankel's function takes the form

$\Psi_{r}(h)=C\left(z-z_{2}-i \frac{\pi}{2} \zeta\right)$ for $z<z_{2}$,

where $C$ is an arbitrary coefficient. Matching Eqs. (20) and (24) within the common region $z_{1}<z<z_{2}$ implies that $C=i \omega A_{0}$, then the modal equation reads

$\gamma_{l}=\frac{z_{2}+i \zeta \pi / 2}{z_{1}-z_{c}\left(1-1 / \varepsilon_{c}\right)-i \zeta \pi / 2}$.

It becomes convenient here to use the altitude coordinate $h=z-z_{c}$ (Fig. 1) while referring to atmospheric profiles. Using the above relation $4 \mu_{0} \omega \sigma_{2} \zeta^{2}=1$, we may link both boundaries through the scale height, as follows

$h_{2}=h_{1}+\zeta \ln \frac{1}{4 k^{2} \zeta^{2}}$.

As a consequence, Eq. (25) involves only five unknown parameters of the cavity, namely: (i) the thickness of the icy crust $z_{\mathrm{c}}$, (ii) the real part of the crust permittivity $\operatorname{Re} \varepsilon_{c}$, (iii) its loss tangent $\delta$, assumed to be weak enough such as $\delta \ll \operatorname{Re} \varepsilon_{c}$, (iv) the altitude of the conduction boundary $h_{1}$, and (v) the scale height of the conductivity $\zeta$. Considering $\zeta \ll h_{1}$ and merging Eq. (25) with the expression of $\gamma_{l}$ in Eq. (10), it is straightforward to derive the approximate modal equations in terms of eigenfrequency and quality factor as

$$
\begin{aligned}
\omega_{l}= & \frac{c}{a}\left[l(l+1) \frac{h_{1}+z_{C} / \operatorname{Re} \varepsilon_{c}}{h_{1}+z_{c}+\zeta \ln \left(1 / 4 k^{2} \zeta^{2}\right)}\right]^{1 / 2} \\
& \times\left[1-i\left(\frac{z_{C} \delta / \operatorname{Re} \varepsilon_{c}+\pi \zeta / 4}{h_{1}+z_{C} / \operatorname{Re} \varepsilon_{c}}+\frac{\pi \zeta / 4}{h_{1}+z_{c}+\zeta \ln \left(1 / 4 k^{2} \zeta^{2}\right)}\right)\right],
\end{aligned}
$$

$$
\begin{aligned}
Q & =\left|\frac{\operatorname{Re} \omega}{2 \operatorname{Im} \omega}\right| \\
& =\left[\frac{2 z_{C} \delta / \operatorname{Re} \varepsilon_{c}+\pi \zeta / 2}{h_{1}+z_{C} / \operatorname{Re} \varepsilon_{c}}+\frac{\pi \zeta / 2}{h_{1}+z_{c}+\zeta \ln \left(1 / 4 k^{2} \zeta^{2}\right)}\right]^{-1}
\end{aligned}
$$

One may check that for $z_{c}=0$ the above expressions reduce to the usual ones for the terrestrial SR (Füllekrug, 2000). It is worth emphasizing the crucial contribution of crustal parameters for controlling the Titan's SR eigenmodes, which makes a fundamental difference with the Earth's SR. In the next sections we take advantage of this particularity for constraining the cavity parameters.

\section{PWA observations and constrained model cavity}

The average profiles of atmospheric electron conductivity and $36 \mathrm{~Hz}$ signal strength measured with the PWA-MIP-RP-ELF instruments (Hamelin et al., 2007; López-Moreno et al., 2008; Béghin et al., 2009) are plotted in Fig. 2, in middle-left and middle-right panels, respectively. For the purpose of comparison we have included a few additional measurements. The first one (left panel) is the zonal wind speed profile obtained with the Doppler Wind Experiment (DWE), conducted with the Green Bank and Parkes Radio Telescopes (Bird et al., 2005), plotted along with the Huygens Probe descent velocity. The last right panel plots are the raw and smoothed tilt variations of the Huygens vertical axis derived from the DISR experiment (Karkoschka et al., 2007). Prior to proceeding with the analysis of PWA data we address the efficiency of the ELF emission mechanism for further comparison with the strength of the Huygens signal.

\subsection{Modulation mechanism of the current source}

The current-driven ion-acoustic instability mechanism presumed to be the ionospheric source of Titan's SR (Béghin et al., 2007), also called two-stream instability, was known long ago to develop in space and laboratory collisional plasmas (e.g. Bychenkov et al., 1994). The instability is due to the anomalous conductivity that occurs as soon as the relative drift velocity between electrons and positive ions exceeds the local sound speed, and the ELF modulation bandwidth stretches up to the ion-plasma frequency (Appendix B.1). Since the modulated currents are draped around Titan's ram hemisphere (Ledvina and Cravens, 1998), they 
Altitude (km)

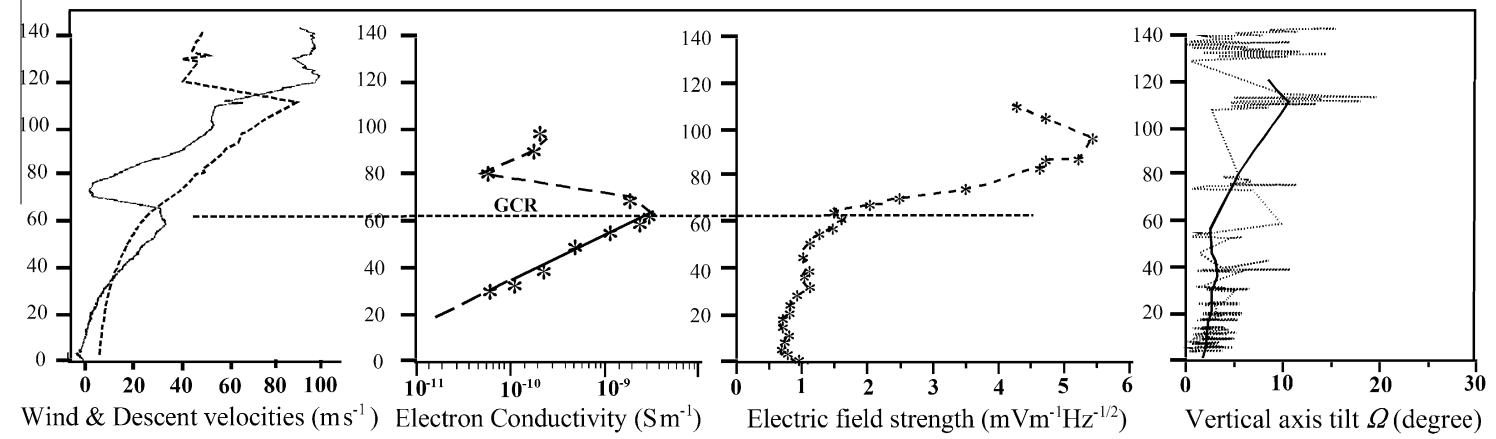

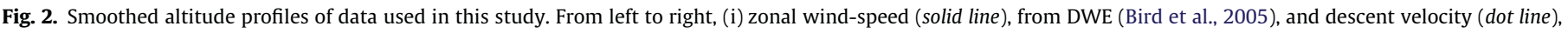
from the mission event list; (ii) average electron conductivity from PWA-MIP-RP; (iii) smoothed field strength of the 36 Hz line from PWA-ELF; (iv) raw and smoothed profiles

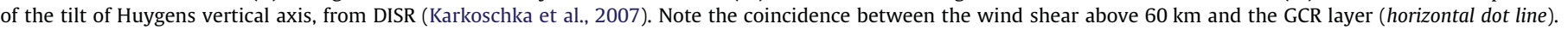

may be compared to vibrating strings sustaining the resonance of the cavity.

On Earth, the natural excitation of the ion-acoustic instability has been observed locally in the equatorial electrojet by rocket borne instruments in form of electrostatic wave at $25 \mathrm{~Hz}$ with $2 \mathrm{mV} \mathrm{m}^{-1}$ amplitude (Pfaff et al., 1987). Likewise, the current modulation mechanism was proved to be an efficient stimulus for ELF waves with an active experiment carried out in the region of the terrestrial polar electrojet (Mc Carrick et al., 1990), using the High-Power Auroral Stimulation (HIPAS) HF (High Frequency) heating facility, near Fairbanks, Alaska. In this experiment we assume that the generation of ELF waves was coherently stimulated by forcing the anomalous conductivity of the medium with deposition of modulated powerful HF waves, in presence of the polar electrojet. Electromagnetic ELF waves $(6-76 \mathrm{~Hz})$ with magnetic field amplitudes of up to $2 \mathrm{pT}$ and $0.2 \mathrm{mV} \mathrm{m}^{-1}$ electric field were received at the ground, $35 \mathrm{~km}$ from the HIPAS site. Considering that the E region illuminated with the HF source was covered by a large number of sidelobes distributed uniformly in azimuth over $45^{\circ}$ (Mc Carrick et al., 1990), the extent of the disturbed region was roughly $90 \mathrm{~km}$, to be compared to about $60^{\circ}$ of latitudinal extent $(\sim 2800 \mathrm{~km})$ for Titan's current sheets. Applying the geometrical scale factor 90:2800, one deduces the transfer ratio 2:62 pT that will be considered further in Section 5.4. Notice that this ratio is probably underestimated, because the HIPAS fixed frequency stimulus was coherent over $90 \mathrm{~km}$, contrary to random noise of Titan's ion-acoustic turbulence.

\subsection{Mode degree and subsurface parameters constrained by PWA observations}

If we assume that Titan is an ideal Schumann cavity with a nominal radius of $2575 \mathrm{~km}$, introducing $\gamma_{1}=1$ in Eq. (10), the first three eigenfrequencies are found roughly $26,45,64 \mathrm{~Hz}$ for $l=1$, 2,3 respectively. The frequencies predicted by models are either close to these values (Nickolaenko et al., 2003) or widely spread around, depending on the cavity structure (Simões et al., 2007). Thus, the first parameter to be confirmed is the degree $l$ of the $36 \mathrm{~Hz}$ signal. Assuming that the coefficient $\gamma_{l}$ is constant for all modes, introducing $f=36 \mathrm{~Hz}, l=2$ in Eq. (10) with $a=2600 \mathrm{~km}$ including partly the dielectric crust contribution, we get $\gamma_{l}=1.56$. From Eq. (25) it appears that $\gamma_{l}$ cannot be lower than unity since $z_{2}>z_{1}$ by definition (Fig. 1). Thus $36 \mathrm{~Hz}$ cannot be the fundamental eigenmode. However, this frequency could be either the second or the third mode (Simões et al., 2007). Thus, we need additional data to determine unambiguously the mode degree. It is a common feature of exponential scale-height ionospheric models (e.g., Sentman, 1990b), that both horizontal and radial electric components reach maximum amplitudes at around the conduction boundary $h_{1}$. One can see in Fig. 2 that the maximum strength of the $36 \mathrm{~Hz}$ signal lies just below $100 \mathrm{~km}$ altitude. Therefore, whatever combination of electric field components might have been measured by PWA antenna (Section 5.3), we may constrain $h_{1}$ to lie at around $100 \mathrm{~km}$.

The third constraining parameter is the quality factor $Q \sim 6$ (Fig. 3, left-hand panel) derived from an average of 61 individual ELF power spectra with 16 frequency bins each, measured between the altitudes of $90.7-80.5 \mathrm{~km}$, where the $36 \mathrm{~Hz}$ signal strength is maximum. Although this value is comparable to the terrestrial ones (e.g., Chand et al., 2009), it is almost one order of magnitude larger than predicted by Titan's models with a perfectly conductive ground (Nickolaenko et al., 2003), whereas $Q$ values as large as 5 are possible in the presence of a perfectly conductive surface beneath a crust $100 \mathrm{~km}$ thick (Simões et al., 2007). Thus, introducing $Q=6$ in the modal Eq. (28) allows us to cross-constrain the remaining four unknown parameters, namely: the thickness $z_{\mathrm{c}}$ of the crust, its complex permittivity ( $\operatorname{Re} \varepsilon_{\mathrm{c}}$ and loss tangent $\delta$ ), and the conductivity scale height $\zeta$ of the upper boundary. The loci the matrix solutions are plotted in Fig. 4 for the two options $l=2$ and 3, as a function of the scale-height (horizontal axis) and the crust permittivity (vertical axis) for different values of crust thickness.

The only available measurement of the surface permittivity in the ELF range comes from the PWA-MIP data at the Huygens landing site. The sounding area embodied about one meter depth and a first estimate led to $\operatorname{Re} \varepsilon_{c} \sim 1.8$, subsequently constrained between 1.9 and 3.2 (Grard et al., 2006). In the microwave range of the Cassini Radar Scatterometer, the mean ground permittivity of Titan's surface is $\operatorname{Re} \varepsilon_{c}=2.2 \pm 0.05$ (Wye et al., 2007). However, it is not obvious that the electric characteristics of the crust should be the same at depth, because the ice seems to be contaminated by deposits of organic residue such as tholins (Zarnecki et al., 2005). In a crust made of water Ice I (Tobie et al., 2005) with chemical inclusions such as ammonia concentration up to $10 \%$, the permittivity might reach 3-4 (Lorenz, 1998), and may vary with increasing temperature at depth (Section 6.2). Assuming an unlikely wide range of crust permittivity $2<\operatorname{Re} \varepsilon_{c}<5$, and ionospheric conductivity scale height $3<\zeta<15 \mathrm{~km}$, for $l=3$ we find that the thickness of the crust should be $400-800 \mathrm{~km}$ (Fig. 4, left panel). This is much larger than the upper bound $(\sim 160 \mathrm{~km})$ predicted by recent models (Deschamps et al., 2010). Moreover, even for models with unrealistic low amount of radioactive elements in the silicate fraction (e.g., Grasset and Sotin, 1996), the presence of ammonia within the crust is not compatible with such thickness because a low eutectic temperature leads to partial melt and formation of an ocean at a maximum depth of about $100 \mathrm{~km}$. Thus, the mode $l=3$ is incompatible with a frequency of $36 \mathrm{~Hz}$ and a quality factor of 6 . On the contrary, for $l=2$, a permittivity $2<\operatorname{Re} \varepsilon_{c}<4$ and loss 

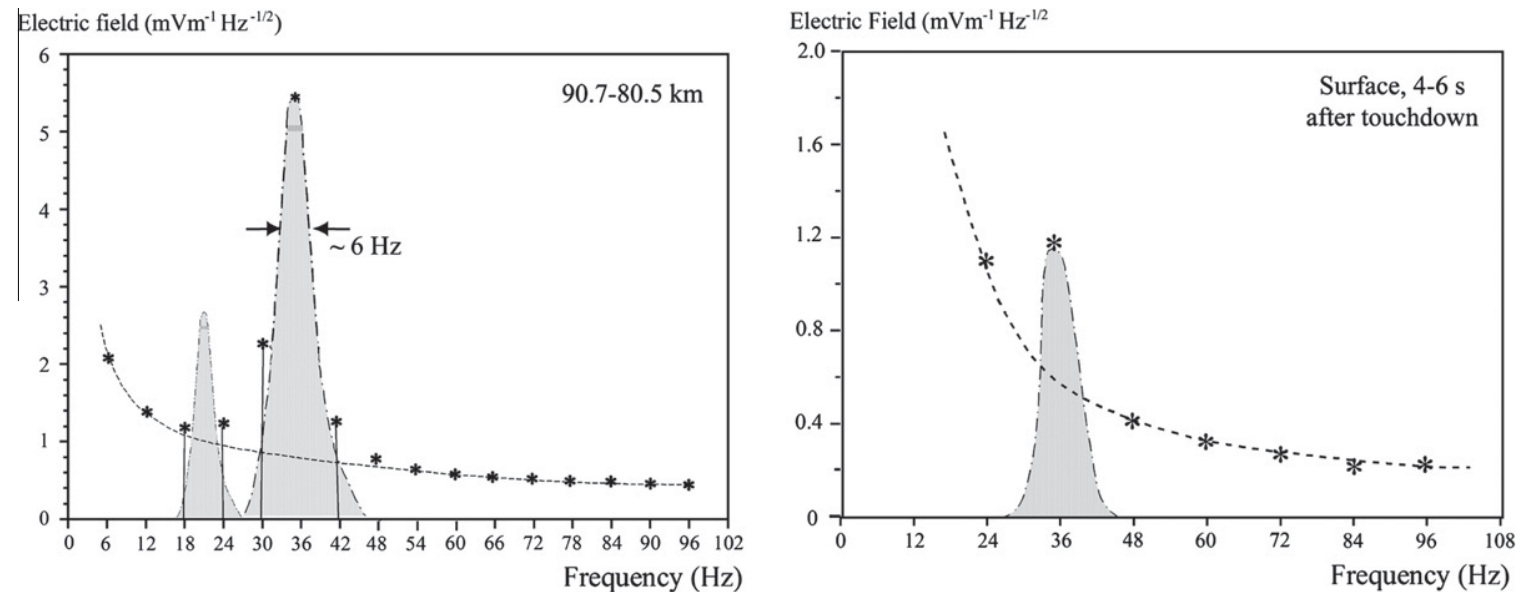

Fig. 3. Asterisks, left panel: average of 61 PWA-ELF power spectra data in $3 \mathrm{~Hz}$ resolution mode, without the missing Channel A odd bins [(2n - 1$) 3$ ]. Right panel: power spectrum (even bins $[(2 n) 6] \mathrm{Hz}$ ) transmitted $8 \mathrm{~s}$ after touchdown in $6 \mathrm{~Hz}$ resolution mode. Dot lines: smoothed background noise. Shaded areas: power spectra expected to have been observed with nominal frequency resolution. Vertical solid lines in left panel: quadratic mean composite of expected side band values plus noise fitting the measurements (asterisks).
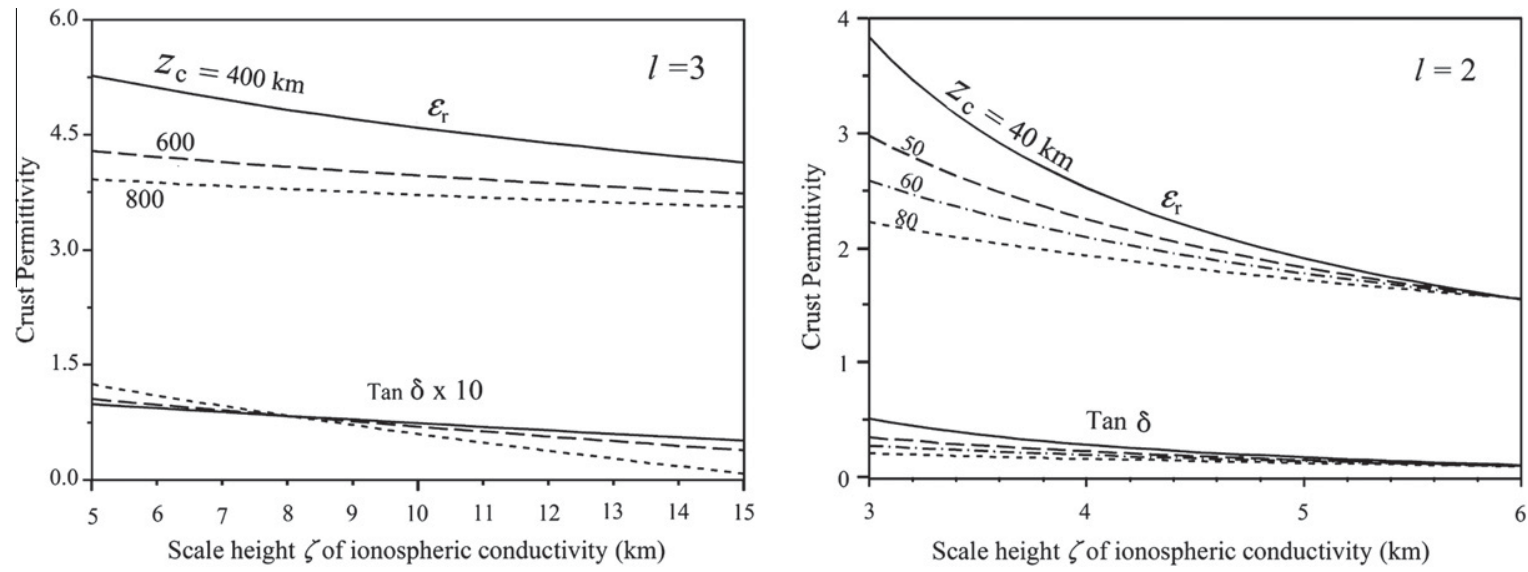

Fig. 4. Loci of cross-constrained crustal parameters versus the ionospheric scale-height for eigenmodes $l=3$ (left panel) and $l=2$ (right panel).

tangent $0.25<\delta<0.5$ (i.e., a conductivity $\sigma=1$ to $4 \mathrm{nS} \mathrm{m}^{-1}$ ), the crust might be $40-80 \mathrm{~km}$ thick, and the ionospheric conductivity scale-height between 3 and $4 \mathrm{~km}$ (Fig. 4, right panel). Thus, the $36 \mathrm{~Hz}$ frequency fulfils the required conditions to be the second eigenfrequency of Titan's SR, denoted below as an $\mathrm{LSM}_{2}$ mode.

\subsection{Altitude profiles of field components of the $\mathrm{LSM}_{2}$ mode}

Using the formalism developed in Sections 3 and 4, our purpose was to check whether the constrained parameters of the cavity are compatible with the height profile of the PWA signal strength. The first step of the procedure consisted in computing the field profiles for a few case-studies considering the most likely values of the constrained parameters. The second step consisted in retrieving the profiles of the electric signals that would have been collected by the PWA antenna, and to compare afterwards the retrieved profiles with the measurements. The model field profiles are computed integrating the scalar potential Eq. (A7) through successive slabs $5 \mathrm{~km}$ thick along the vertical path outlined in Fig. 1. At each interface we apply the transfer conditions Eq. (17) taking care of their analytic continuity within the complex plane, namely, the sudden phase shift at the ground-atmosphere interface mentioned in Section 4. The normalization factor $\left(\omega A_{0}\right)$ is given an arbitrary value of $1 \mathrm{Vm}^{-1}$, while $A_{r}$ is kept constant up to the vicinity of $z_{2}$.
The field amplitudes are derived from Eqs. (8a) and (8b) using the usual spherical harmonic function

$Y(\theta, \varphi)=P_{l}^{m}(\cos \theta) \cos m \varphi$,

and its derivatives, where $P_{l}^{m}$ are Legendre polynomials of degree $l$ and order $m$, the latter being an integer or zero. The terrestrial vertical current sources are suited to traditional SR modes which are characterized by a single azimuthal magnetic component and $m=0$ (Sentman, 1990b; Nickolaenko and Sentman, 2007). On the contrary, Titan's horizontal current sheets (Fig. 5) constrain $m$ to be an integer (Wait, 1962). We must, therefore, consider the tesseral modes $\operatorname{LSM}_{l}^{m}$ to denote Titan's SR, with $m=1$ for the fundamental order. For $l=2$, the Legendre polynomial of first order reads $P_{2}^{1}=-3 / 2 \sin 2 \theta$. Introducing this expression in Eq. (29) we obtain

$$
\begin{aligned}
& Y(\theta, \varphi)=-\frac{3}{2} \sin 2 \theta \cos \varphi, \\
& \frac{\partial Y}{\partial \theta}=-3 \cos 2 \theta \cos \varphi ; \frac{\partial Y}{\partial \varphi}=\frac{3}{2} \sin 2 \theta \sin \varphi,
\end{aligned}
$$

where $\theta$ and $\varphi$ are the colatitude and the longitude of the observer with respect to the source.

Without loss of generality, we consider the configuration plotted in Fig. 5, where the source and the observer lie within a reference system with an ideal north-south axis orientation of 


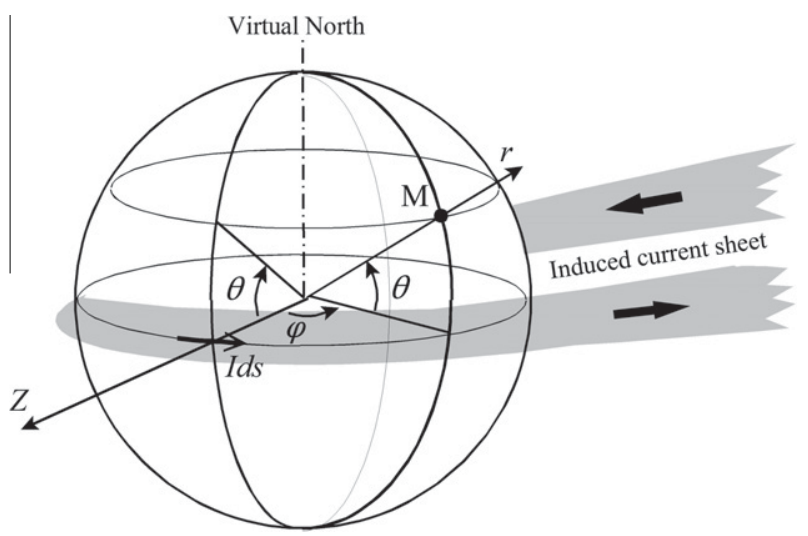

Fig. 5. Spherical coordinates for arbitrary observer's location (M) with respect to a horizontal elementary current dipole Ids, in the ideal configuration of azimuthal current sheet.

the co-rotating Saturn's dipolar magnetospheric plasma flow (Ledvina and Cravens, 1998). For better understanding of the differences between Titan's horizontal and Earth's vertical current sources, the expressions of $Y(\theta, \varphi)$ for the modes $l=1,2$, and $m=1,0$ respectively, are reported in Table 1 . It is seen that the antinodes (peak values) of the radial electric component for the second Titan's tesseral mode $\mathrm{LSM}_{2}^{1}$ take place at latitudes $\pm 45^{\circ}$ $\left( \pm 180^{\circ}\right)$ with respect to the source region, contrary to the Earth's $\mathrm{TEM}_{2}^{0}$ zonal mode, where the antinodes lie at $0^{\circ}$ and $180^{\circ}$.

Introducing Eq. (30) in the field expressions Eqs. (8a) and (8b), the numerical integration of Eq. (17) was performed by steps of $5 \mathrm{~km}$ starting from $z=0$ up to $z_{2}$ (Fig. 1), for constrained parameters derived from Fig. 4 (right panel) and different values of $\theta, \varphi$. A few case-studies for $z_{\mathrm{c}}$ ranging between 40 and $80 \mathrm{~km}$ are reported in Table 2, and the retrieved field profiles for case-study \#3 are plotted in Fig. 6. With the exception of the weak contribution of the GCR layer, the altitude profiles of the horizontal components are similar to those of the terrestrial two-scale-height model (Sentman, 1990b). The slope of the horizontal electric component $\left(\left|E_{h}\right|\right)$ profile does not change substantially across the ground surface, reflecting the fact that the conductivity of the crust is low in this example $\left(\tan \delta=0.27\right.$, i.e., $\sigma=1.34 \mathrm{nS} \mathrm{m}^{-1}$ ). However, the profile of the radial electric field component exhibits a different behavior with respect to Earth's conditions. The amplitude of $E_{r}$ rises suddenly across the ground surface (Fig. 6) while the permittivity drops from $\varepsilon_{c}$ to 1 , as it can be checked by substituting the interface relationship for $\partial \varphi / \partial z$ given by Eq. (17) in the expression of $E_{r}$ in Eq. (8b).

\subsection{Retrieved amplitude profile of the measured electric field}

The next step consisted in retrieving the Huygens measurements obtained with the above model field profiles. The PWA electric antenna was made of two short monopoles, each $45 \mathrm{~cm}$ long, mounted on two opposite sides of the gondola (Grard et al., 2006), yielding an effective length of about $1.6 \mathrm{~m}$ along the Huygens horizontal axis (Cadène, 1995). Such conditions imposed by severe constraints of the mission are obviously not comparable with several meters long vertical antennas installed onboard Earth's balloon flights (Ogawa et al., 1979). However, since the response to electromagnetic fields of a spinning horizontal antenna is attitude dependent, the modulation of the $36 \mathrm{~Hz}$ signal has been revealed by a statistical analysis of time intervals comparable to the spin period (15-35 s) of the Huygens gondola (Béghin et al., 2009). In the present study, in order to reveal the global contribution of all electric components associated with the variations of the tilt of Huygens vertical axis, we have computed the signal strength profiles that should have been measured in the presence of model field profiles such as those plotted in Fig. 6 .

The measured signal strength is the root mean square of horizontal $E_{h}$ and radial $E_{r}$ components projected along the antenna, which reads

$$
E_{M}=\left[\left(E_{h} \cos \Omega\right)^{2}+\left(E_{r} \sin \Omega\right)^{2}\right]^{1 / 2} \quad \text { with } \quad \Omega=\left[1-\cos ^{2} \eta \cos ^{2} \tau\right]^{1 / 2}
$$

where $\Omega$ is the smoothed tilt value (Fig. 2, right panel), computed from values of the roll $(\eta)$ and pitch $(\tau)$ angles (Table 1, in Karkoschka et al., 2007). The retrieved profiles, corresponding to three case-studies $(\# 2,3,4)$ from Table 2 , are plotted along with the experimental profile in Fig. 7. While the altitude $h_{1}$ of conductivity threshold has been prescribed at $100 \mathrm{~km}$ in the model, the peak values of retrieved responses are observed at $95 \pm 5 \mathrm{~km}$ (i.e., one step of the model), fitting closely the spread of experimental values. This satisfactory model-data fit allows us to confirm the altitude of $h_{1}$ at around $100 \mathrm{~km}$ for the model of atmospheric conductivity discussed in Section 5.5.

Note that the retrieved profiles in Fig. 7 have been plotted using a homothetic transformation scale in order to match their peak amplitude with the experimental value, so that the scale ratio yields a rough estimate of the absolute field amplitudes plotted in Fig. 6. It appears from Eq. (31) that the main contribution to the $36 \mathrm{~Hz}$ signal measured at around $90 \mathrm{~km}$ comes from the dominant component $E_{r}$ while the average tilt plotted in Fig. 2 (right panel) at this altitude is about $9^{\circ}$. Substituting the latter value and the measured amplitude $\left|E_{M}\right|=5.5 \mathrm{mV} \mathrm{m}^{-1} \mathrm{~Hz}^{-1 / 2}$ in Eq. (31), neglecting the amplitude of $E_{h}$ which is 40 times smaller than $E_{r}$ at this altitude (Fig. 6), we obtain an estimate of the power spectral density of the radial electric component $\left|E_{r}\right| \sim 35 \mathrm{mV} \mathrm{m}^{-1} \mathrm{~Hz}^{-1 / 2}$ at $90 \mathrm{~km}$. This value must be compared with the arbitrary amplitude of $1600 \mathrm{mV} \mathrm{m}^{-1}$ (Fig. 6) obtained with the normalization factor $\omega A_{0}=1 \mathrm{Vm}^{-1}$. According to the experimental calibration ratio between sine amplitude and power spectral density, applying the homothetic ratio $(35: 1600)$ to all components plotted in Fig. 6, the amplitude of the horizontal electric component $E_{h} \mid$ is found $\sim 1 \mathrm{mV} \mathrm{m}^{-1}$ at around $h_{1}$, and the horizontal magnetic induction

Table 1

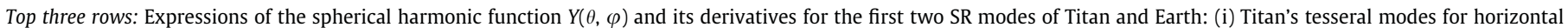

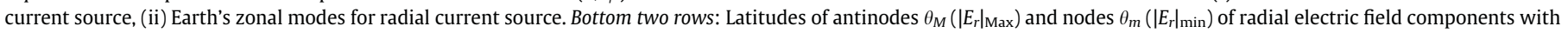
respect to the source location.

\begin{tabular}{|c|c|c|c|c|}
\hline & \multicolumn{2}{|c|}{ Titan's tesseral modes } & \multicolumn{2}{|c|}{ Traditional Earth's zonal modes } \\
\hline & $\operatorname{LSM}_{1}^{1}(l=1, m=1)$ & $\operatorname{LSM}_{2}^{1}(l=2, m=1)$ & $\operatorname{TEM}_{1}^{0}(l=1, m=0)$ & $\operatorname{TEM}_{2}^{0}(l=2, m=0)$ \\
\hline$Y(\theta, \varphi)$ & $-\sin \theta \cos \varphi$ & $-3 \sin \theta \cos \theta \cos \varphi$ & $\cos \theta$ & $1 / 2\left(3 \cos ^{2} \theta-1\right)$ \\
\hline$\partial Y(\theta, \varphi) / \partial \theta$ & $-\cos \theta \cos \varphi$ & $-3 \cos 2 \theta \cos \varphi$ & $-\sin \theta$ & $-3 \sin \theta \cos \theta$ \\
\hline$\partial Y(\theta, \varphi) / \partial \varphi$ & $\sin \theta \sin \varphi$ & $3 \sin \theta \cos \theta \sin \varphi$ & 0 & 0 \\
\hline$\theta_{M}\left(\left|E_{r}\right|_{\operatorname{Max}}\right)$ & $\pm 90^{\circ}$ & $\pm 45^{\circ}\left( \pm 180^{\circ}\right)$ & $0^{\circ}, 180^{\circ}$ & $0^{\circ}, 180^{\circ}$ \\
\hline$\theta_{m}\left(\left|E_{r}\right|_{\min }\right)$ & $0^{\circ}, 180^{\circ}$ & $0^{\circ}, 180^{\circ}$ & $\pm 90^{\circ}$ & $\pm 54.7^{\circ}\left( \pm 180^{\circ}\right)$ \\
\hline
\end{tabular}


Table 2

Case-studies of Titan's cavity parameters constrained by the plots in Fig. 4 (right panel).

\begin{tabular}{|c|c|c|c|c|c|c|c|c|c|c|c|}
\hline Parameters & $z_{\mathrm{c}}(\mathrm{km})$ & $\operatorname{Re} \varepsilon_{\mathrm{c}}$ & $\tan \delta$ & $h_{1}(\mathrm{~km})$ & $\zeta_{0-60}(\mathrm{~km})$ & $\zeta_{60-80}(\mathrm{~km})$ & $\zeta_{80-h 2}(\mathrm{~km})$ & $h_{2}(\mathrm{~km})$ & $\sigma_{2}\left(\mathrm{Sm}^{-1}\right)$ & $\theta$ (deg) & $\varphi$ (deg) \\
\hline \multicolumn{12}{|l|}{ Case-study } \\
\hline \#1 & 40 & 3 & 0.37 & 100 & +5.2 & -5.2 & 3.5 & 136 & $7 \times 10^{-5}$ & 50 & 20 \\
\hline \#2 & 50 & 2.65 & 0.295 & 105 & +5.2 & -5.2 & 3.7 & 143 & $6.4 \times 10^{-5}$ & 40 & 45 \\
\hline \#3 & 50 & 2.48 & 0.27 & 100 & +5.2 & -5.2 & 3.6 & 136.5 & $6.8 \times 10^{-5}$ & 40 & 45 \\
\hline$\# 4$ & 50 & 2.25 & 0.23 & 95 & +5.2 & -5.2 & 4 & 141 & $5.5 \times 10^{-5}$ & 40 & 45 \\
\hline \#5 & 60 & 2.1 & 0.195 & 100 & +5.2 & -5.2 & 4 & 141 & $5.5 \times 10^{-5}$ & 50 & 30 \\
\hline \#6 & 80 & 2 & 0.17 & 100 & +5.2 & -5.2 & 3.7 & 138 & $6.4 \times 10^{-5}$ & 45 & 10 \\
\hline
\end{tabular}

$z \quad$ Altitude $(\mathrm{km})$

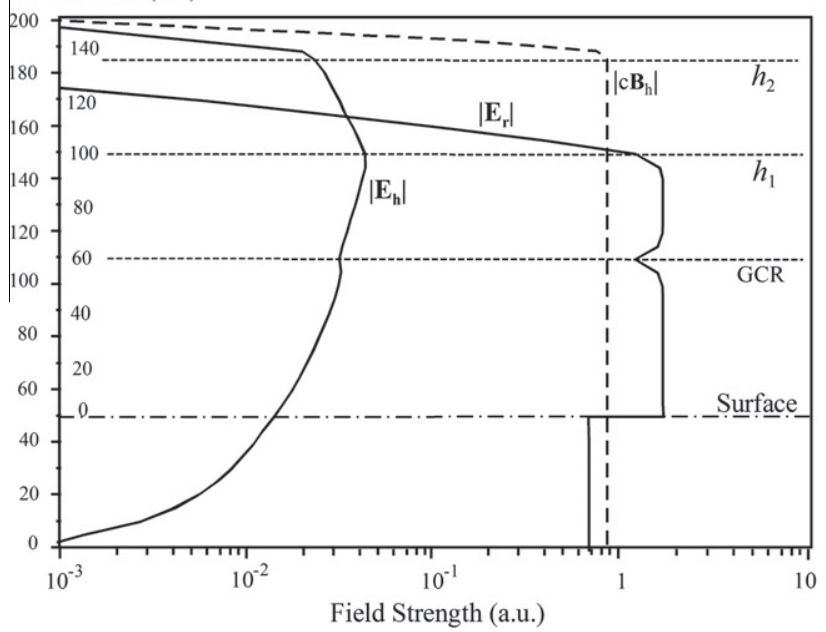

Fig. 6. Normalized electric and magnetic field profiles for the case-study \#3 in Table 2. Field strength units are for $\omega A_{0}=1\left(\mathrm{Vm}^{-1}\right)$. Amplitudes of horizontal electric and magnetic fields are root mean square of $\theta$ and $\varphi$ components.

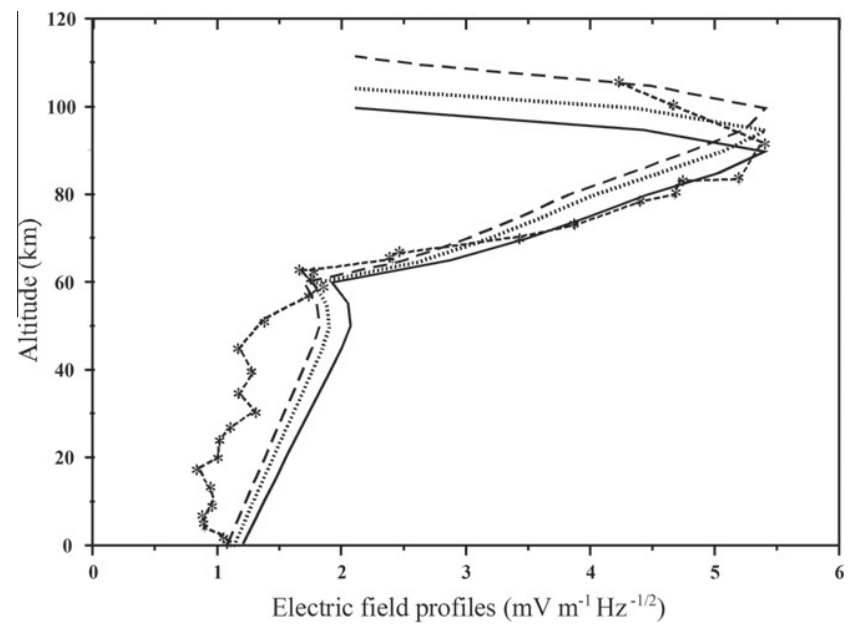

Fig. 7. Comparison between measured (asterisks and interpolated line) and retrieved electric field strength assumed to be collected by the PWA antenna for three casestudies \#2, 3 and 4 from Table 2 (dash, dot, and solid lines, respectively). The retrieved profiles are normalized in order to match their peak amplitude with the experimental value.

$\sim 62$ pT. Such surprisingly high values are nearly two orders of magnitude larger than the usual values for the Earth's SR, as pointed-out by Simões et al. (2007). Using the geometric scale factor derived in Section 5.1 from the stimulated ELF emissions performed by Mc Carrick et al. (1990), we saw that a magnetic induction of $62 \mathrm{pT}$ would be necessary indeed at the top of the cavity to feed the SR mode. Then, the source current-density according to the expression $\mu_{0} J=B$ would be about $50 \mu \mathrm{Am}^{-1}$. The latter value is equivalent to a current sheet of $150 \mathrm{~A}$ through $60^{\circ}$ in longitude $(\sim 2800 \mathrm{~km})$, which is roughly $0.1 \%$ of the global current induced by the Kronian magnetospheric plasma flow (Béghin et al., 2007).

\subsection{Constrained model of the upper ionosphere conductivity}

Since the atmospheric conductivity profile is seen to control Titan's SR modes, the models built up in Section 5.3 are assumed to involve global azimuthal and latitudinal extents, well beyond the region of Huygens descent. Therefore, we were forced to release some of constraints imposed by the local measurements of conductivity by PWA instruments above the GCR layer in favor of the constrained parameters derived in Section 5.3 and subsequently introduced in model profiles such as the one plotted in Fig. 8 (solid line). However, although the retrieved field profiles plotted in Fig. 7 match satisfactorily the experimental one, we have so far no measurement of electron conductivity in the global atmosphere up to the upper boundary $h_{2}$ to confirm or dispute our model profiles. Thus, we were obliged to resort to theoretical models.

Sharp variations of conductivity above $100 \mathrm{~km}$ are thought to be linked to haze stratifications distributed in mid latitude regions, with scale heights of few kilometers, such as those revealed by far-infrared remote sensing from the Cassini CIRS, the Cassini Composite Infrared Spectrometer experiment (de Kok et al.,

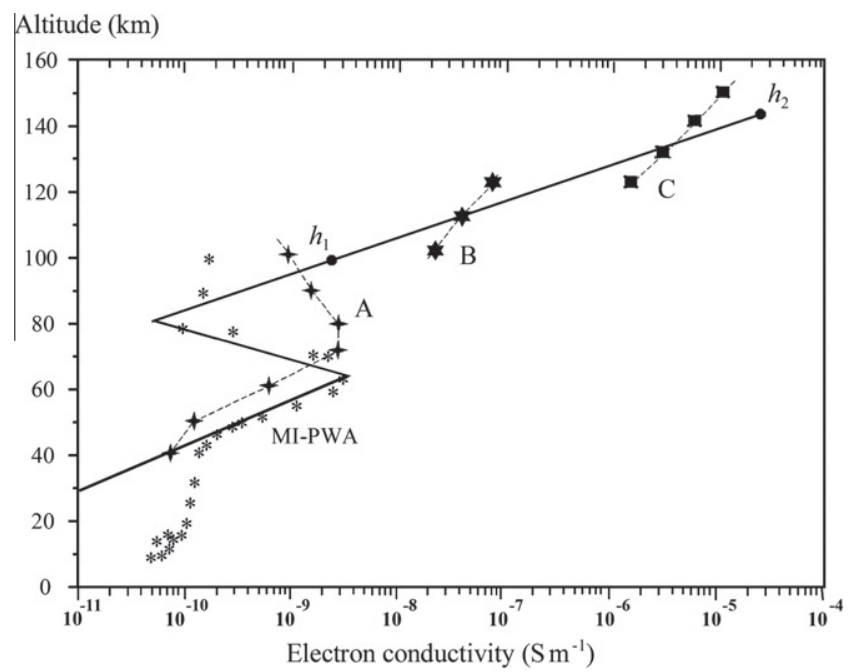

Fig. 8. Attempts in matching measured and predicted electron conductivity profiles, for case-study \#5 with parameters from Tables 2 and 3. Asterisks: averaged PWA-MIP-RP measurements up to $100 \mathrm{~km}$. Crosses: Model A, with predominating GCR ionization, this work. Solid line: model used for retrieval of profiles plotted in Fig. 7. Stars: Model B, with predominating solar photoemission threshold of $6.6 \mathrm{eV}$, after Borucki and Whitten (2008). Squares: Model C, same as Model B, but photoemission threshold $6 \mathrm{eV}$. 
Table 3

Ionospheric parameters used for the conductivity profiles models A, B and C in Fig. 8.

\begin{tabular}{|c|c|c|c|c|c|c|c|c|c|c|c|}
\hline \multirow[t]{2}{*}{ Altitude (km) } & \multirow[t]{2}{*}{ Temp. (K) } & \multirow{2}{*}{$\begin{array}{l}\text { Solar attenuation } \\
\text { (at } 250 \mathrm{~nm} \text { ) }\end{array}$} & \multirow{2}{*}{$\begin{array}{l}\text { GCR ionization } \\
\text { rate }\left(\mathrm{cm}^{-3} \mathrm{~s}^{-1}\right)\end{array}$} & \multicolumn{2}{|c|}{ Photoemission threshold $(\mathrm{eV})$} & \multicolumn{3}{|c|}{ Particle size $(\mu \mathrm{m})$} & \multicolumn{3}{|c|}{ Number density $\left(\mathrm{m}^{-3}\right)$} \\
\hline & & & & B & $\mathrm{C}$ & A & B & C & A & B & C \\
\hline 40 & 70.66 & $5.32 \times 10^{-8}$ & 0.45 & n.a. & n.a & 3.4 & n.a. & n.a. & $5.0 \times 10^{6}$ & n.a. & n.a. \\
\hline 50 & 71.33 & $4.42 \times 10^{-7}$ & 0.50 & n.a. & n.a. & 3.4 & n.a. & n.a. & $5.0 \times 10^{6}$ & n.a. & n.a. \\
\hline 60 & 76.87 & $3.67 \times 10^{-6}$ & 1.00 & n.a. & n.a. & 3.4 & n.a. & n.a. & $5.0 \times 10^{6}$ & n.a. & n.a. \\
\hline 70 & 103.94 & $3.04 \times 10^{-5}$ & 3.02 & n.a. & n.a. & 3.4 & n.a. & n.a. & $5.0 \times 10^{6}$ & n.a. & n.a. \\
\hline 80 & 123.61 & $2.53 \times 10^{-4}$ & 10.02 & n.a. & n.a. & 3.4 & n.a. & n.a. & $5.0 \times 10^{6}$ & n.a. & n.a. \\
\hline 90 & 134.90 & $1.79 \times 10^{-3}$ & 12.00 & n.a. & n.a. & 3.4 & n.a. & n.a. & $4.3 \times 10^{6}$ & n.a. & n.a. \\
\hline 100 & 142.06 & $9.62 \times 10^{-3}$ & 14.00 & 6.6 & n.a. & 3.4 & 0.9 & n.a. & $3.7 \times 10^{6}$ & $1.2 \times 10^{7}$ & n.a. \\
\hline 110 & 147.31 & $4.06 \times 10^{-2}$ & 10.79 & 6.6 & n.a. & n.a. & 0.9 & n.a. & n.a. & $1.2 \times 10^{7}$ & n.a. \\
\hline 120 & 151.00 & $1.40 \times 10^{-1}$ & 8.31 & 6.6 & 6.0 & n.a. & 0.9 & 0.9 & n.a. & $1.2 \times 10^{7}$ & $1.2 \times 10^{7}$ \\
\hline 130 & 156.34 & $4.03 \times 10^{-1}$ & 6.43 & n.a. & 6.0 & n.a. & n.a. & 0.9 & n.a. & n.a. & $1.2 \times 10^{7}$ \\
\hline 140 & 158.55 & $9.99 \times 10^{-1}$ & 4.95 & n.a. & 6.0 & n.a. & n.a. & 0.9 & n.a. & n.a. & $1.2 \times 10^{7}$ \\
\hline 150 & 161.92 & 1.00 & 3.82 & n.a. & 6.0 & n.a. & n.a. & 0.9 & n.a. & n.a. & $1.2 \times 10^{7}$ \\
\hline 160 & 163.77 & 1.00 & 2.60 & n.a. & 6.0 & n.a. & n.a. & 0.9 & n.a. & n.a. & $1.2 \times 10^{7}$ \\
\hline
\end{tabular}

2007). According to modeling of Titan's atmosphere electric properties (Borucki and Whitten, 2008), it appears that the key factors controlling the conductivity profile between the GCR layer and about $150 \mathrm{~km}$ are haze structures of aerosols, whereas little is known about size, abundance, photoemission, and electrophyllic properties of these particles (Tomasko and West, 2009). Measurements of the IRIS infrared imager onboard Voyager 1 (Smith et al. 1981) suggested that highly scattered particles, almost certainly condensed organics, with radii between 1 and $5 \mu \mathrm{m}$ and mole fraction $10^{-8}-10^{-6}$ such as nitrile condensates, are presumably permeating the stratosphere in aerosol clouds at altitudes of $58-90 \mathrm{~km}$ (Mayo and Samuelson, 2005). However, the most advanced models do not account for such thin and irregular structures (Borucki and Whitten, 2008). In the latter work, the authors consider particles with downward constant mass flux of $4 \times 10^{-13} \mathrm{~kg} \mathrm{~m}^{-2} \mathrm{~s}^{-1}$ from an altitude of $150 \mathrm{~km}$ or above, down to low altitude regions. The particle mass density is supposed to be $500 \mathrm{~kg} \mathrm{~m}^{-3}$, i.e. similar to that of tholin aerosols coated with liquid methane $\left(420 \mathrm{~kg} \mathrm{~m}^{-3}\right)$ or ethane $\left(545 \mathrm{~kg} \mathrm{~m}^{-3}\right)$.

Stimulated by the encouraging results of Borucki and Whitten (2008), predicting electron conductivities at $140 \mathrm{~km}$ compatible with our constrained values, we tried to identify which parameters might be adjusted within superimposed step-by-step structures, in order to fit an average profile constrained by the SR model. The results are plotted in Fig. 8 with the parameters of the case-study \#5 in Table 2, and three portions (A, B, and C) of profiles with the parameters defined in Table 3 . The model $A$, from low altitude up to $100 \mathrm{~km}$, appears globally to account quite satisfactorily for the PWA-MIP-RP measurements of electron-ion conductivities up to the GCR layer (Hamelin et al., 2007; López-Moreno et al., 2008), except the sharp decrease at around $75 \mathrm{~km}$. In model A we have considered particles with large aggregate surface radii of $3.4 \mu \mathrm{m}$ (Lavvas et al., 2010), and GCR being the main source of ionization (Table 3). Models B and C above $100 \mathrm{~km}$ are excerpts from Borucki and Whitten (2008) for particles with different photo ionization thresholds. Although further refinements could be conceivable, the above estimate allows us to check that our constraints applied to the upper atmospheric conductivity could be met. We did not succeed, however, to simulate the deep decrease of conductivity at around $75 \mathrm{~km}$ which appears linked to the zonal wind shear (Fig. 2), as it falls out of scope of this article, although some related aspects are discussed in next Section 6.1.

\section{Discussion}

Among the results of this work, the inferred subsurface structure is undeniably the one that is challenging most the models of Titan interior. This point is addressed hereunder in Section 6.2, while experimental aspects relevant to the specific behavior of the PWA experiment are discussed in Appendix B. Concerning the atmosphere, we investigate what kind of physical processes could possibly account for the apparent correlation between the data from independent instruments, as plotted in Fig. 2. Because the tilt of Huygens vertical axis with respect to the zenith seems to be somewhat correlated with the zonal wind and to have a presumed influence on the behavior of PWA boom antenna, we discuss the relevant points in Appendix B (Appendix B.3), while involved physical processes are analysed here below.

\subsection{Could a correlation between profiles of Huygens tilt, zonal wind and conductivity be explained?}

The DWE Team has always been wary of the coincidence (M.K. Bird, University of Bonn, private communication) that Huygens descended through a surprisingly low-wind region at heights $100-60 \mathrm{~km}$ (Fig. 2 left panel), from a few tens of seconds to several minutes after the parachute exchange. In previous studies, it was not completely excluded that abnormal parachute dynamics during this phase might possibly be responsible for the apparent drop in the zonal wind speed (Bird et al., 2005). On the basis of measurements of independent instruments, it is proposed here that such an artifact is quite unlikely. First, we may rule out any significant link between the Huygens vertical axis tilt and the average conductivity profile measured by PWA-MIP-RP. Although the mutual impedance measurement is influenced to the first-order by the projection of the descent velocity vector along the Huygens vertical axis $X_{p}$ (Hamelin et al., 2007), second-order effects due to tilt variations smaller than $10^{\circ}$ would be undetectable. Thus, the correlation between the profiles of conductivity and zonal wind is not tilt dependent. Secondly, we do not see any possibility for the tilt being directly caused by the presence of aerosols.

There is a good correlation, however, between the wind and tilt data (Dzierma et al., 2007). If one knows the wind and descent speed as a function of altitude, with the probe parameters one can predict how much and how long the probe would tilt wherever the wind is not constant with altitude. We found that this predic tion is pretty close to the observations under the large parachute, at altitudes above $110 \mathrm{~km}$. Under the small parachute, at altitudes below $110 \mathrm{~km}$, there is still a small correlation between the expected and observed tilt, but there is also another large component of the tilt, which is almost certainly the one-second swing when the probe-parachute system oscillates in a scissor-like mode (Karkoschka et al., 2007). Although the relatively low value of the long time-scale tilt $\left(\Omega<5^{\circ}\right)$ was satisfactorily modeled using the AGC data from transmission Channel B between Huygens and Cassini (Dzierma et al., 2007), the fast oscillations were not 
expected before the mission and had not been simulated. The latter authors suggested that these oscillations could have been generated by the probe, and then were picked up by the parachute some fraction of a second later, as for instance if one or more parachute risers were kinked by wind gusts or temporarily tangled. Wind and tilt are thus somewhat correlated, but not perfectly. These observations favor the conclusion that the process responsible for the large-scale Doppler wind profile, more especially the drop at $75 \mathrm{~km}$, is most likely of atmospheric origin, as it is suggested below.

Concerning the zonal wind profile itself, two reports have been subsequently published (Folkner et al., 2006; Flasar et al., 2010) after the initial publication of the DWE measurements by Bird et al. (2005). Except for an interesting suggestion by Walterscheid and Schubert (2006) that atmospheric gravitational tides could be responsible for decelerating the superrotating atmosphere at the Huygens site, there is still no clear identification of the agent responsible for the wind speed drop to nearly zero at $75 \mathrm{~km}$ (M.K. Bird, University of Bonn, private communication). The mechanism suggested by Walterscheid and Schubert (2006) might explain the formation of thin and sharp horizontal haze layers assumed to be produced by vertical transport of aerosol particles driven by Saturn gravitational tides. According to the standard model of superrotating (eastward) zonal wind in equatorial and polar regions considered by these authors, the tide-induced vertical wavelength may become comparable to thin layers of aerosol concentration a few $\mathrm{km}$ thick, at a critical transition region between 90 and $100 \mathrm{~km}$ altitude. It is then plausible that similar sharp transitions in mid latitude regions could correspond to steep gradients of retrieved haze extinction profiles of infrared spectra observed by the Cassini CIRS experiment (de Kok et al., 2007). Thus, such layering mechanisms, producing compaction and rarefaction structures of aerosol concentration in the vicinity of the ionospheric boundaries $h_{1}$ and $h_{2}$, should be considered in further development of conductivity profile models, as suggested in Section 5.5.

\subsection{Constrained depth of the buried liquid water ocean and Titan's interior models}

The second major constraint derived from our analysis is the thickness of the subsurface icy crust (Section 5.2). Assuming the lower conductive layer of the cavity to be the crust-ocean interface, let us summarize the implications of a crust $40-80 \mathrm{~km}$ thick. Sotin et al. (2009) demonstrated that different models support such a thickness range whether internal heat is transferred either by conduction or by convection. After describing the consequences for each case, we will draw the subsequent inferences on heat flux, mantle temperature, and crust composition. If heat is transferred by conduction and assuming no lateral variations, the conservation of heat can be written

$\frac{\partial}{\partial r}\left(\kappa \frac{\partial T}{\partial r}\right)+\frac{2 \kappa}{r} \frac{\partial T}{\partial r}=\rho C_{p} \frac{\partial T}{\partial t}-H$

where $\kappa$ is the thermal conductivity of the crustal material, $r$ is the radial distance, $\rho$ the density, $C_{p}$ the specific heat, $T$ the temperature and $H$ the internal volumetric heating rate. This equation is solved with the following assumptions: (i) the thermal conductivity of the crust is that of pure ice, (ii) the crust is in thermal equilibrium with the internal heat, and (iii) spherical geometry can be neglected because the thickness of the crust is small compared to the radius. With these conditions, the heat flux through the crust is constant. The thermal conductivity of ice strongly depends on temperature as $\kappa=0.4685+488.12 / T$, which can be approximated at better than $5 \%$ by $\kappa=570 / T$ in the involved temperature range $90-273 \mathrm{~K}$. Note that the thermal conductivity varies from $\kappa=6.33 \mathrm{~W} \mathrm{~m}^{-1} \mathrm{~K}^{-1}$ at the surface $\left(T_{0}=92 \mathrm{~K}\right)$ to $\kappa=2.11 \mathrm{~W} \mathrm{~m}^{-1} \mathrm{~K}^{-1}$ at the melting temperature of pure ice. The $1 / T$ variation leads to the following analytic solution of Eq (32):

$\ln \left(\frac{T}{T_{0}}\right)=\frac{\xi}{z_{c}} \ln \left(\frac{T_{w}}{T_{0}}\right)$ and $Q_{t}=4 \pi a_{0}^{2} q=4 \pi a_{0}^{2} \frac{570}{z_{c}} \ln \left(\frac{T_{w}}{T_{0}}\right)$,

where $\xi=a_{0}-r$ is the depth, $z_{c}$ is the crust thickness (here between 40 and $80 \mathrm{~km}), a_{0}$ is the Titan radius, $T_{0}$ the surface temperature, $T_{w}$ the temperature of the ocean at the base of the ice crust, $q$ and $Q_{t}$ are the heat flux and the total escaping heat power from Titan surface, respectively. The heat to be transferred through the ice crust is the internal heat produced by the decay of radioactive elements contained in the rock fraction. This value can be debated, but one can take end-members corresponding to the amount in enstatite chondrites and carbonaceous chondrites (e.g. Grasset et al., 2000). These end-member values provide heat power between $400 \mathrm{GW}$ and $650 \mathrm{GW}$ (Fig. 9). If the temperature at the base of the crust is the melting temperature of pure ice $\left(T_{w}=260 \mathrm{~K}\right)$, then the heat which can be released is larger than the maximum value of $650 \mathrm{GW}$ for values of the crust thickness up to $80 \mathrm{~km}$. Therefore, the present values of $40-80 \mathrm{~km}$ do not support an ocean temperature of $260 \mathrm{~K}$. Note that this value is less than the $273 \mathrm{~K}$ at room pressure because the melting temperature of ice decreases with increasing pressure. However, the temperature of the ocean can be lower, because ammonia, a strong anti-freezing compound, is likely to be present in Titan's ocean (Tobie et al., 2005). With an ocean temperature of $200 \mathrm{~K}$, the equilibrium total heat power is smaller than $650 \mathrm{GW}$ for values of the crust thickness larger than $55 \mathrm{~km}$ (Fig. 9). The temperature at the crust-ocean interface has to be as low as $160 \mathrm{~K}$ in order to get a value of $650 \mathrm{GW}$ with a $40 \mathrm{~km}$ thick crust. The constrained range of the crust thickness (40-80 km) would be therefore consistent with a crust conduction profile over an ammonia-rich ocean with the amount of ammonia decreasing (i.e., ocean temperature increasing) with increasing thickness.

The internal heat may also be transferred by convection processes if the buoyant forces overcome the viscous forces. As shown by previous studies (e.g. Grasset and Sotin, 1996), convection in Titan's crust can operate when the thickness of the ice layer becomes larger than a critical depth (Fig. 10). This critical depth depends on the viscosity of ice at the ocean-crust interface: the smaller the viscosity, the smaller the critical depth. With a typical viscosity of $10^{14} \mathrm{~Pa}$ s for ice at its melting point, the critical value of the crust thickness is only $15 \mathrm{~km}$. The critical viscosity is equal to $2 \times 10^{15} \mathrm{~Pa} \mathrm{~s}$ and $2 \times 10^{16} \mathrm{~Pa} \mathrm{~s}$ for a crust thickness $40 \mathrm{~km}$ and

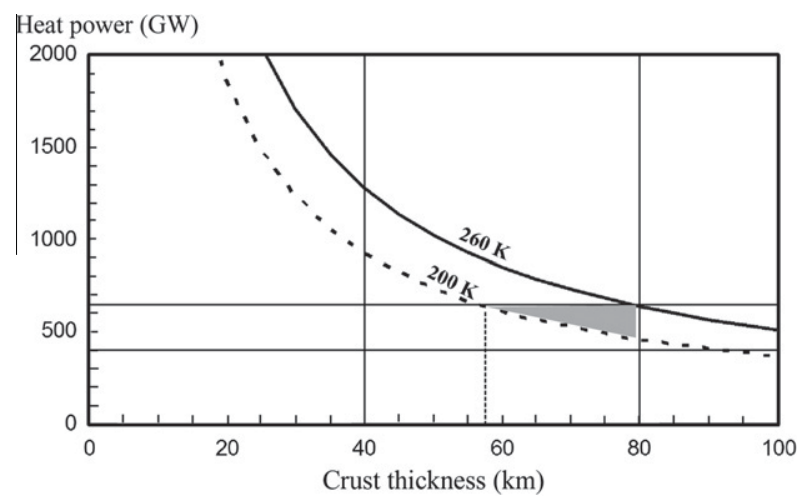

Fig. 9. Equilibrium heat power with respect to the crust thickness for two values of the temperature at the crust-ocean interface. The thin vertical solid lines are the upper and lower bounds of the crust thickness constrained by the SR. The two horizontal lines represent end-member values of the internal radioactive power. 


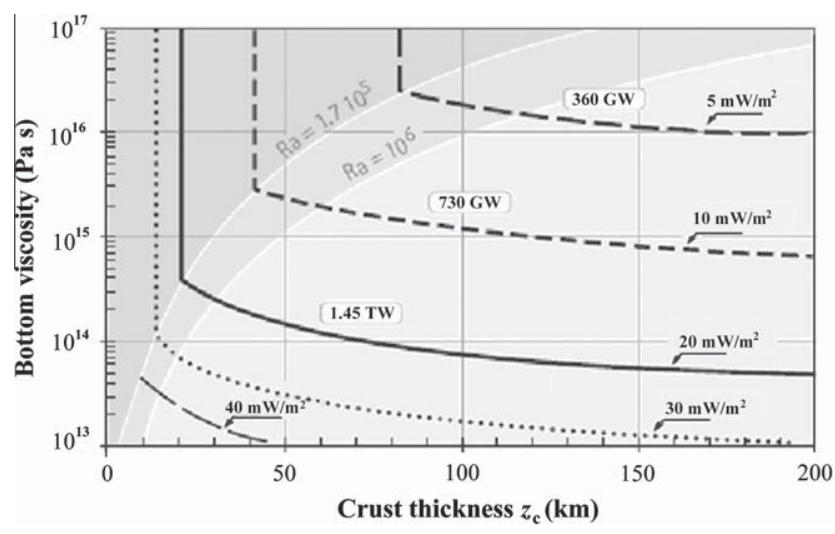

Fig. 10. Ice viscosity at the crust/ocean interface as a function of the crust thickness The dark grey area corresponds to a domain where heat is transferred by conduction (excerpt from Sotin et al., 2009).

$80 \mathrm{~km}$, respectively. Two other elements must be kept in mind. First, there are uncertainties on the value of the ice viscosity at a given temperature, and the viscosity at the melting temperature can vary between $10^{13}$ and $10^{15} \mathrm{~Pa}$. Second, a Titan's primordial large eccentricity implies that there was little dissipation in the interior, which yields viscosity larger than $10^{15} \mathrm{~Pa}$ s at present time (Tobie et al., 2005). With such a value of viscosity, a thickness of $40 \mathrm{~km}$ would imply a heat power much larger than $600 \mathrm{GW}$ (Fig. 10). On the other hand, a thickness of $80 \mathrm{~km}$ is consistent with a heat power between 450 and $600 \mathrm{GW}$ and suggests viscosity at the base of the crust between $3 \times 10^{15}$ and $2 \times 10^{16} \mathrm{~Pa}$ s. The proposed low bound of $40 \mathrm{~km}$ seems therefore difficult to reconcile with a convective model, unless additional heat has to be transferred such as latent heat released as the ice crust thickens. Moreover, the upper bound of $80 \mathrm{~km}$ is consistent with a convective model in equilibrium with radioactive internal heating. Assuming that the viscosity law follows an Arrhenius-like law with an activation energy of $50 \mathrm{~kJ} / \mathrm{mole}$ and a typical viscosity of $10^{14} \mathrm{~Pa} \mathrm{~s}$ at the melting point of pure ice, the viscosity range $3 \times 10^{15}-2 \times 10^{16} \mathrm{~Pa}$ s at the crust-ocean interface translates into a temperature range $235-220 \mathrm{~K}$, which is in agreement again with the presence of ammonia in the ocean.

The Cassini observations have not yet provided definitive answers regarding the question about whether convection or conduction is the heat transfer mode within the icy crust. For example, the presence of ${ }^{40} \mathrm{Ar}$ in Titan's atmosphere (Niemann et al., 2005) cannot be explained if conduction has operated for several billion years. On the other hand, since ${ }^{40} \mathrm{Ar}$ comes from the decay of ${ }^{40} \mathrm{~K}$ contained in the silicates of the core, there must be some exchange between the interior and the atmosphere through convection processes. One may also explain the presence of ${ }^{40} \mathrm{Ar}$ in the atmosphere during a global resurfacing event that occurred some hundred of million years ago (Tobie et al., 2005). Another fact is the absence of evidence for cryo-volcanism at present time. It does not prove the absence of convection processes, since other planetary bodies, like Mars, are likely to undergo convection without expressing it by volcanic activity at present time. In conclusion, it is not possible today to solve the question of convection versus conduction based upon the atmospheric concentration of ${ }^{40} \mathrm{Ar}$, neither the absence of cry-volcanism on Titan's surface.

\section{Summary and conclusion}

From an analytic development of mathematical and physical properties of the postulated Titan's SR, in significant progress with respect to previous works (Béghin et al., 2007, 2009, 2010), we derived a model which fits satisfactorily the Huygens PWA-ELF field profile measurement. The specificity of Titan's cavity, with a layered inhomogeneous structure and ionospheric current sources instead of lightning, makes a significant difference with respect to the Earth's traditional SR. Taking advantage of this specificity, in spite of severe technical limitations of the PWA experiment, the model allows us to constrain the main physical parameters of both ionospheric and subsurface boundaries of the cavity. The icy-crust properties constrained by this study suggest a quasi-solid sheet with finite viscosity overlaying an ammonia-rich ocean, which is consistent with convection or conduction processes propagating through it. It appears that a crust thickness larger than $80 \mathrm{~km}$ would be unstable to convection processes unless the viscosity at the base of the crust would be larger than $2 \times 10^{16}$ Pa s (Fig. 10), and the crust-ocean interface temperature less than $260 \mathrm{~K}$. On the other hand, a convective $40 \mathrm{~km}$ thick crust cannot be in equilibrium with the internal heating, suggesting that the crust should continue to grow at the expense of the ocean. Our conclusion is that there is, either a $\sim 55 \mathrm{~km}$ thick conductive crust with a crust-ocean interface temperature of $\sim 200 \mathrm{~K}$ and a crust viscosity larger than $5 \times 10^{15}$ Pa s (Fig. 10), or an $80 \mathrm{~km}$ thick convective crust with an interface temperature of $220-235 \mathrm{~K}$ and a maximum viscosity of $\sim 2 \times 10^{16}$ Pa s (Figs. 9 and 10). Although each of those two possibilities of heat transfer would comply with our model of the Titan's SR, it is shown (Section 5.2 and Fig. 4) that a better estimate or further measurements of both crust permittivity and atmospheric conductivity profile, would allow to reduce accordingly the range of uncertainty.

After Huygens results and those obtained during the first phase of the Cassini mission, we must now wait for long term exploration of the Saturn system in order to progress with the question of the crust and ocean depth. Unless, in the meantime, the extended Cassini mission until July 2017 allows advanced measurements of Titan's gravity field. It is known indeed that accurate measurements of the gravity field might provide the value of the degree 2 Love number $k_{2}$, a coefficient of the periodic gravitational potential (Sotin et al., 2009). Since this number is very sensitive to the presence of a liquid layer at depth, with expected values as large as 0.4 compared to values of $10^{-2}$ when there is no liquid shell (Castillo et al., 2000), we may expect from this method, either a confirmation that a water ocean is present at depth, or otherwise, a challenge to our interpretation of Titan's SR.

\section{Acknowledgments}

C.B. is very grateful to Michael Bird for providing us with firsthand information about the Doppler Wind Experiment, and would like to acknowledge the fruitful discussions with Frederic Deschamps, Jonathan Lunine, Jean-Pierre Lebreton and Stefan Schröder during the preparation of this paper. Members from LATMOS-IPSL were supported for this experiment by the Centre National d'Etudes Spatiales under contract 60015. This work was partly performed (C.S.) at the Jet Propulsion Laboratory, California Institute of Technology. C.S. acknowledges support by the JPL Research and Technology Development Program and the NASA Outer Planets Research Program. F.S. is supported by an appointment to the NASA Postdoctoral Program at the Goddard Space Flight Center, administered by the Oak Ridge Associated Universities. Authors are very grateful to the two reviewers, including Dr. A.P. Nickolaenko, who carefully reviewed this work, allowing us to substantially improve the manuscript.

\section{Appendix A. Helmholtz equations of Titan's LSM modes}

While the wave equation is established in its general form (Eq. (5)), no special relationship is imposed between both potentials $A$ 
and $\Psi$. Introducing the condition of radial magnetic field component to be zero for TEM modes (Galejs, 1972), and assuming the vector potential to have three independent components such as $A_{r}, A_{\theta}, A_{\varphi}$, the radial component of the first equation in Eq. (3) reads

$\mu_{0} H_{r}=\frac{1}{r \sin \theta}\left[\frac{\partial}{\partial \theta}\left(A_{\varphi} \sin \theta\right)-\frac{\partial A_{\theta}}{\partial \varphi}\right]=0$.

Applying the principle of variable separation, it is straightforward that the unique solution of Eq. (A1) implies that both components $A_{\theta}$, and $A_{\varphi}$ are identically null. Thus, the vector potential is radial and may be written as a scalar function $A=A_{r}(r) Y(\theta, \varphi)$, where the spherical harmonic function $Y(\theta, \varphi)$ is defined in terms of Legendre polynomials in Section 5.3. Similarly, the scalar potential reads $\Psi=\Psi_{r}(r) Y(\theta, \varphi)$. Developing each spherical component in Eq. (5), yields

$\hat{\mathbf{r}} \Rightarrow\left[\frac{\nabla \times \nabla \times \mathbf{r} A_{r} Y(\theta, \varphi)}{r}\right]-i \frac{k^{2} n^{2}}{\omega} \frac{\partial \Psi_{r}}{\partial r}+k^{2} n^{2} A_{r} Y(\theta, \varphi)=0$,

$\hat{\boldsymbol{\theta}} \Rightarrow \frac{\partial A_{r}}{\partial r}-i \frac{k^{2} n^{2}}{\omega} \Psi_{r}=0 ; \hat{\varphi} \Rightarrow \frac{\partial A_{r}}{\partial r}-i \frac{k^{2} n^{2}}{\omega} \Psi_{r}=0$.

We note that both latitudinal and azimuthal components in Eq. (A3) lead to the same relationship between $A_{r}$ and $\Psi_{r}$. This relation is reminiscent of a gauge condition between both potentials. However it is not the conventional Lorentz gauge introduced occasionally as a complementary condition (e.g., Sentman, 1990b) that reads as

$\nabla \cdot A-i \frac{k^{2} n^{2}}{\omega} \Psi_{r}=0$

where the divergence operator replaces the radial derivative in Eq. (A3). A gauge condition between both potentials might be arbitrarily chosen as long as no peculiar condition is imposed (Nisbet, 1955). However, since we imposed radially polarized modes with $H_{r}=0$, the "gauge-like" relation given by Eq. (A3) derives directly from Eq. (5), and then implicitly from the Maxwell equations as shown by Bahar and Fitzwater (1983, Appendix A). Developing the first member of Eq. (A2) yields

$$
\begin{aligned}
& {\left[\frac{\nabla \times \nabla \times r A_{r} Y(\theta, \varphi)}{r}\right]} \\
& \quad=\frac{1}{r^{2}}\left[-\frac{1}{\sin \theta} \frac{\partial}{\partial \theta}\left(\sin \theta \frac{\partial A_{r} Y}{\partial \theta}\right)-\frac{1}{\sin ^{2} \theta} \frac{\partial^{2} A_{r} Y}{\partial \phi^{2}}\right],
\end{aligned}
$$

which is known as the angular Laplacian of the scalar function $A_{r}(r)$ $Y(\theta, \varphi)$, and may be developed into spherical harmonics function (Groemer, 1996) as

$\left[\frac{\nabla \times \nabla \times \mathbf{r} A_{r} Y(\theta, \varphi)}{r}\right]=\frac{l(l+1)}{r^{2}} A_{r} Y(\theta, \varphi)$,

where $Y(\theta, \varphi)$ is defined by Eq. (30) in Section 5.3. Combining Eqs. (5) and (A6) yields a second height-gain differential equation between both potentials, in some way symmetrical to Eq. (A.3) although independent of it

$\frac{\partial \Psi_{r}}{\partial r}=i \omega\left[1-\frac{l(l+1)}{k^{2} r^{2} n^{2}}\right] A_{r}$.

Using both height-gain Eqs. (A3) and (A7) and substituting successively $A_{r}$ then $\Psi$ we obtain two independent second order differential Helmholtz equations for the SR Titan's modes

$\frac{\partial^{2} \Psi_{r}}{\partial z^{2}}+k^{2}\left(n^{2}-\frac{l(l+1)}{k^{2} a^{2}}\right) \Psi_{r}+\frac{l(l+1)}{k^{2} a^{2} n^{2}-l(l+1)}\left(n^{2} \nabla n^{-2}\right) \frac{\partial \Psi_{r}}{\partial z}=0$,

$\frac{\partial^{2} A_{r}}{\partial z^{2}}+k^{2}\left(n^{2}-\frac{l(l+1)}{k^{2} a^{2}}\right) A_{r}+\left(n^{2} \nabla n^{-2}\right) \frac{\partial A_{r}}{\partial z}=0$, in which we made the change of variable $z=r-a+z_{c}$, where $a$ is the mean Titan's radius and $z_{\mathrm{c}}$ the crust thickness (Fig. 1). Moreover, in the derivation of these equations we made use of the thin shell approximation (Galejs, 1972) which leads to consider $k^{2} r^{2} \approx k^{2} a^{2}$ as a constant as far as the mean radius of the cavity is much larger than its depth.

\section{Appendix B. Experimental open questions}

\section{B.1. Detection of other eigenmodes}

Here we address the question as to why the PWA instrument did not detect any other resonance than the second eigenmode at $36 \mathrm{~Hz}$ (Section 5.1), although a faint signal above random noise of the power-spectrum bin at around $48 \mathrm{~Hz}$ (Fig. 7c in Grard et al. (2006)), might possibly be a fleeting signature of the mode $l=3$. A plausible cause for the absence of highest order modes could be that the Huygens descent latitude $(\theta)$ would occur for minimum values of the spherical harmonic function (Eq. (29)) and its derivative, leading to field amplitudes smaller than the background noise. Alternatively, a most likely explanation implies that the upper cutoff frequency of the ion-acoustic instability (Section 5.1), i.e., the plasma frequency of positive ions, would lie at around $40-50 \mathrm{~Hz}$ in the source region. The ion-plasma frequency obeys the relation

$f_{i}=\frac{e}{2 \pi}\left(\frac{n_{i}^{+}}{m_{i} \varepsilon_{0}}\right)^{1 / 2}$

where $n_{i}^{+}$and $m_{i}$ are the positive ions density and mass, respectively. Assuming that the best conditions for an efficient modulation of the ionospheric currents would occur within $200-400 \mathrm{~km}$ altitude range (Béghin et al., 2009), with an electron-ion density between $10^{6}$ and $10^{7} \mathrm{~m}^{-3}$ (Borucki et al., 1987, 2006) and a mean ion mass between 17 and $170 \mathrm{amu}$, the cut-off frequency would be $40-50 \mathrm{~Hz}$, then, ruling out accordingly the source of SR higher modes.

However, the above process cannot explain the absence of the fundamental mode $(l=1)$. It is seen from Eq. (25) that the coefficient $\gamma_{l}$ is roughly proportional to the ratio $z_{2} / z_{1}$. On Earth, the values of the coefficient $\gamma_{l}$ of first two modes are such as $\gamma_{1} / \gamma_{2} \sim 1.08$. As the radial thickness of Titan's cavity is about twice as large as that of Earth, this ratio might even be smaller, say $\sim 1.07$. Since the coefficient $\gamma_{l}$ for the second mode has been prescribed as $\gamma_{2}=1.56$ (Section 5.2), assuming the ratio $\gamma_{1} / \gamma_{2}$ lying between unity and 1.07, the maximum range for $\gamma_{1}$ is then 1.56 to1.67. Substituting these values in Eq. (10), the fundamental mode $f_{1}$ is found between 20.1 and $20.8 \mathrm{~Hz}$. We recall that, due to the loss of telemetry channel A (Lebreton et al., 2005), only two spectral bins (18 and $24 \mathrm{~Hz}$ ) among those that might possibly contain $f_{1}$ were transmitted to Earth in the highest resolution telemetry mode (Fig. 3, left panel). Thus, since $f_{1}$ lies within the $3 \mathrm{~dB}$ bandwidth $(21 \pm 1.5 \mathrm{~Hz})$ of the missing power-spectral bin, its contribution could not be observed in the data set, unless overlapping the adjacent bins at 18 and $24 \mathrm{~Hz}$ significantly above the ambient noise.

It is seen from Eq. (28), that the quality factor for $l=1$ would be roughly the same as for $l=2$, yielding a $3.5 \mathrm{~Hz}$ half-power bandwidth, that could overlap the adjacent bins with some $3 \mathrm{~dB}$ sideband damping. Applying to the fundamental mode the same retrieval procedure as for the second harmonic (Section 5.4), keeping the same parameters in Table 2 and a constant value for $A_{0}$ (Section 5.1 ), we find that the fundamental mode would be damped by about $6 \mathrm{~dB}$ compared to mode $l=2$. Adding the noise level of bins $18-24 \mathrm{~Hz}$ (Fig. 3, left panel), the sideband contributions of a $21 \mathrm{~Hz}$ resonance would be then damped by $6+3 \mathrm{~dB}$ with respect to its 
peak value. Thus, such marginal contribution of fundamental Titan's SR could not be seen in the transmitted data.

\section{B.2. Measurements after landing}

Another challenging issue is why the $36 \mathrm{~Hz}$ signal disappeared $16 \mathrm{~s}$ after the Huygens Probe landing. The last significant power spectrum (Fig. 3, right panel), was transmitted about $8 \mathrm{~s}$ after touchdown that occurred at $T_{0} \sim 8869.80 \mathrm{~s}$ Mission Time (Zarnecki et al., 2005). The plot in Fig. 3 is an average power spectrum of two sample ELF electric field waveforms collected and processed onboard about 4 and $6 \mathrm{~s}$ respectively after $T_{0}$. Further measurements performed $8 \mathrm{~s}$ later and transmitted $16.575 \mathrm{~s}$ after $T_{0}$, and all successive data, exhibit nothing else than instrumental noise which reflects a non-nominal performance of the antenna device. This fact is corroborated by hard to explain coincidental abnormal data of the Mutual Impedance measurements of surface conductivity during the first $20 \mathrm{~s}$ following touchdown. During the same period, three photometers, as a part of DISR experiment with different fields of view, observed an unexplained variability of the light intensity for $6 \mathrm{~s}$ after $T_{0}$ (Chapter 7.1 in Schröder (2007)).

In short, it seems that Huygens decelerated a fraction of second for about $12 \mathrm{~cm}$ upon impact while the bottom of the gondola was sinking into a soft solid surface (Zarnecki et al., 2005). More precisely, the probe bounced back after the first impact, had a free fall phase again, then impacted once more and slid for a while, before finally coming to rest. The whole sequence lasted slightly less than $4 \mathrm{~s}$; afterwards Huygens remained motionless (Bettanini et al., 2008). Thus, the data plotted in Fig. 3 (right panel), were collected when the Huygens Probe was at rest with its vertical axis tilted by $3^{\circ}$, whilst it was only $1-2^{\circ}$ during the final part of the descent (Karkoschka et al., 2007). The $12 \mathrm{~cm}$ penetration may have been experienced either by the lower dome of the Probe into the soft surface or on ice pebbles that were situated under the dome just before touchdown (Bettanini et al., 2008). Therefore, the final tilting position might possibly be due to a large icy-cobble on the DISR side of Huygens $X_{p}$ axis, like those seen in the HRI and MRI images (Karkoschka et al., 2007). It is then plausible that at least one of the sensors located at the tip of each boom (Hamelin et al., 2007) might have sunk into the ground deeply enough to divide the initial amplitude of $E_{r}$ by the ground permittivity $\operatorname{Re} \varepsilon_{c}$ as seen in Fig. 6 . The signal strength would have then decreased by at least $6 \mathrm{~dB}$ with respect to the records before impact, and hidden below the background noise (Fig. 3, right panel). In either case, the visible $36 \mathrm{~Hz}$ line testifies that the signal was present at Titan's surface at least for $4 \mathrm{~s}$ after touchdown.

\section{B.3. Wind induced vibrations of the PWA boom-antenna}

There was persistent doubt since the earliest investigations that the $36 \mathrm{~Hz}$ signal could be an artifact somehow related to a mechanical resonance of the boom antenna (Béghin et al., 2007). First and foremost, after static and dynamic cryogenic tests performed in 2010, on flight-spare models of boom at ESA/ESTEC facilities, it was unambiguously concluded that no mechanical resonance was observed in the range $36 \pm 10 \mathrm{~Hz}$, at all temperatures between 100 and $300 \mathrm{~K}$. Second, as seen in Fig. 2 (third panel from left), the average signal amplitude decays monotonically between 90 and $55 \mathrm{~km}$, contrary to the zonal wind that drops suddenly from $50 \mathrm{~m} \mathrm{~s}^{-1}$ to nearly zero at around $75 \mathrm{~km}$ and then grows up to $35 \mathrm{~m} \mathrm{~s}^{-1}$ at $60 \mathrm{~km}$ (Fig. 2, left panel). Third, after the drogue parachute deployment (110 km altitude), the Huygens Probe descent velocity decreases continuously down to the surface, contrary to the signal which reaches a peak value at around $90 \mathrm{~km}$ and then decreases linearly until $60 \mathrm{~km}$. We know that the break of slope at $60 \mathrm{~km}$ is correlated with the conductivity profile of the GCR layer (Béghin et al., 2009). This effect is magnified by a concomitant break of slope of the Huygens Probe tilt (Fig. 2, right panel), that increases accordingly the subsequent contribution of the radial electric component (Fig. 7). Thus, although the tilt might be somewhat wind dependent (Section 6.1), this does not imply that the wind be responsible for a mechanical resonance of the booms. Last but not least, since the $36 \mathrm{~Hz}$ signal was still present near the surface while both wind and descent speeds were weaker than 1 and $4.6 \mathrm{~m} \mathrm{~s}^{-1}$, respectively, as well as a few seconds after landing, we can definitely rule out any boom vibration at $36 \mathrm{~Hz}$ triggered neither by wind-blasts or descent velocity, nor by any reproducible process.

\section{References}

Abramowitz, M., Stegun, I.A., 1972. Handbook of Mathematical Functions. Dove Publications, Inc., New York, 1046pp.

Bahar, E., Fitzwater, M., 1983. Propagation of vertically and horizontally polarized waves excited by distributions of electric and magnetic sources in irregular stratified spheroidal structures of finite conductivity-generalized field functions. Can. J. Phys. 61, 113-127.

Béghin, C. et al., 2007. A Schumann-like resonance on Titan driven by Saturn's magnetosphere possibly revealed by the Huygens Probe. Icarus 191, 251-266.

Béghin, C. et al., 2009. New insights on Titan's plasma-driven Schumann resonance inferred from Huygens and Cassini data. Planet. Space Sci. 57, 1872-1888.

Béghin, C., Hamelin, M., Sotin, C., 2010. Titan's native ocean revealed beneath some $45 \mathrm{~km}$ of ice by a Schumann-like resonance. Comptes Rendus Geosci. 342, 425433.

Bettanini, C., Zaccariotto, M., Angrilli, F., 2008. Analysis of the HASI accelerometers data measured during the impact phase of the Huygens Probe on the surface of Titan by means of a simulation with a finite-element model. Planet. Space Sci. $56,715-727$

Bird, M.K. et al., 2005. The vertical profile of winds on Titan. Nature 438. doi:10.1038/nature04060.

Borucki, W.J. et al., 1987. Predictions of the electrical conductivity and charging of the aerosols in Titan's atmosphere. Icarus 72, 604-622.

Borucki, W.J., Whitten, R.C., Bakes, E.L.O., Barth, E., Tripathi, S., 2006. Predictions of the electrical conductivity and charging of the aerosols in Titan's atmosphere. Icarus 181, 527-544

Borucki, W.J., Whitten, R.C., 2008. Influence of high abundances of aerosols on the electrical conductivity of the Titan atmosphere. Planet. Space Sci. 56, 19-26.

Bychenkov, V.Yu., Myatt, J., Rozmus, W., Tikhonchuk, V.T., 1994. Quasi hydrodynamic description of ion-acoustic waves in collisional plasma. Physics of Plasmas, edit. American Institute of Physics, Melville, NY, USA, vols. 1-8. pp. 2419-2429.

Cadène, F., 1995. Méthodologie de mesure de conductivité d'atmosphères planétaires. Ph.D. Thesis, Université Paris 6. 188pp.

Castillo, J., Mocquet, A., Sotin, C., 2000. Détecter la présence d'un océan dans Europe à partir de mesures altimétriques et gravimétriques. C. R. Acad. Sci. 330, 659666.

Chand, R., Israil, M., Rai, J., 2009. Schumann resonance frequency variations observed in magnetotelluric data recorded from Garhwal Himalayan region India. Ann. Geophys. 27, 3497-3507.

Collin, R., 1991. Field theory of Guided Waves, second ed. IEEE Inc. Press, New York, USA, 852pp.

de Kok, R. et al, 2007. Characteristics of Titan's stratospheric aerosols and condensate clouds from Cassini CIRS far-infrared spectra. Icarus 191, 223-235.

Deschamps, F., Mousis, O., Sanchez-Valle, C., Lunine, J.I., 2010. The role of methanol in the crystallization of Titans's primordial ocean. Astrophys. J. 724, 887-894.

Dougherty, M.K. et al., 2004. The Cassini magnetic field investigation. Space Sci. Rev. 114, 331-383.

Dzierma, Y., Bird, M.K., Dutta-Roy, R., Pérez-Ayúcar, M., Plettemeier, D., Edenhofer, P., 2007. Huygens Probe descent dynamics inferred from Channel B signal level measurements. Planet. Space Sci. 55, 1886-1895.

Flasar, F.M., Baines, K.H., Bird, M.K., Tokano, T., West, R.A., 2010. Atmospheric dynamics and meteorology. In: Brown, R.H. et al. (Eds.), Titan from CassiniHuygens. Springer Publishers, pp. 323-352.

Fischer, G., Gurnett, D.A., 2011. The search for Titan lightning radio emissions. Geophys. Res. Lett. 38, L08206. doi:10.1029/2011GL047316.

Folkner, W.M. et al., 2006. Winds on Titan from ground-based tracking of the Huygens Probe. J. Geophys. Res. 111, E07S02. doi:10.1029/2005JE002649.

Fulchignoni, M. et al., 2002. The characterisation of Titan's atmospheric physical properties by the Huygens Atmospheric Structure Instrument (Hasi). Space Sci. Rev. 104, 395-431.

Füllekrug, M., 2000. Dispersion relation for spherical electromagnetic resonances in the atmosphere. Phys. Lett. A 275, 80-89.

Galejs, J., 1972. Terrestrial Propagation of Long Electromagnetic Waves. Pergamon Press, Oxford, NewYork, Toronto, Sydney, 362pp.

Grard, R., Svedhem, H., Brown, V., Falkner, P., Hamelin, M., 1995. An experimental investigation of atmospheric electricity and lightning activity to be performed 
during the descent of the Huygens Probe on Titan. J. Atmos. Terr. Phys. 57, 575585.

Grard, R. et al., 2006. Properties and related physical characteristics of the atmosphere and surface of Titan. Planet. Space Sci. 54, 1124-1136.

Grasset, O., Sotin, C., 1996. The Cooling Rate of a Liquid Shell in Titan's Interior. Icarus 123, 101-112.

Grasset, O., Sotin, C., Deschamps, F., 2000. On the internal structure and dynamics of Titan. Planet. Space Sci. 48, 617-636.

Greifinger, C., Greifinger, P., 1978. Approximate method for determining ELF eigenvalues in the Earth-Ionosphere waveguide. Radio Sci. 13, 831-837.

Groemer, H., 1996. Geometric Applications of Fourier Series and Spherical Harmonics. Cambridge University Press. 344pp. ISBN: 9780521473187. doi:10.2277/0521473187.

Hamelin, M. et al., 2007. Electron conductivity and density profiles derived from the mutual impedance probe measurements performed during the descent of Huygens through the atmosphere of Titan. Planet. Space Sci. 55, 1964-1977.

Karkoschka, E. et al., 2007. DISR imaging and the geometry of the descent of the Huygens Probe within Titan's atmosphere. Planet. Space Sci. 55, 1896-1935.

Lavvas, P., Yelle, R.V., Griffith, C.A., 2010. Titan's vertical aerosol structure at the Huygens landing site: Constraints on particle size, density, charge, and refractive index. Icarus $210,832-842$.

Lebreton, J.P. et al., 2005. An overview of the descent and landing of the Huygens Probe on Titan. Nature 438, 758-764.

Ledvina, S.A., Cravens, T.E., 1998. A three-dimensional MHD model of plasma flow around Titan. Planet. Space Sci. 45, 1175-1191.

López-Moreno, J.J. et al., 2008. Structure of Titan's low altitude ionized layer from the Relaxation Probe aboard HUYGENS. Geophys. Res. Lett. 35, 222104. doi:10.1029/2008GL035338.

Lorenz, R.D., 1998. Preliminary measurements of the cryogenic dielectric properties of water-ammonia ices: Implications for radar observations of icy satellites. Icarus 136, 344-348.

Lorenz, R.D. et al., 2008. Titan's rotation reveals an internal ocean and changing zonal winds. Science 319, 1649-1651.

Mayo, L.A., Samuelson, R.E., 2005. Condensate clouds in Titan's north polar stratosphere. Icarus 176, 316-330.

Mc Carrick, M.J., Sentman, D.D., Wong, A.Y., Wuerker, R.F., Chouinard, B., 1990. Excitation of ELF waves in the Schumann resonance range by modulated HF heating of the polar electrojet. Radio Sci. 25, 1291-1298.

Niemann, H.B. et al., 2005. The abundances of constituents of Titan's atmosphere from the GCMS instrument on the Huygens probe. Nature 438 (1), 1291-1298. doi:10.1038/nature04122.

Nickolaenko, A.P., Besser, B.P., Schwingenschuh, K., 2003. Model computation of Schumann resonance on Titan. Planet. Space Sci. 51, 853-862.

Nickolaenko, A.P., Sentman, D.D., 2007. Line splitting in the Schumann resonance oscillations. Radio Sci. 42, RS2S13. doi:10.1029/2006RS003473.

Nisbet, A., 1955. Hertzian electromagnetic potentials and associated gauge transformations. Proc. R. Soc. Ser. A 231, 250-263.

Ogawa, T., Kozai, K., Kawamoto, H., 1979. Schumann resonances observed with a balloon in the stratosphere. J. Atmos. Terr. Phys. 41, 135-142.

Pfaff, R.F., Kelley, M.C., Kudeki, E., Fejer, B.G., Bake, D., 1987. Electric field and plasma density measurements in the strongly driven daytime equatorial electrojet, 2. Two-stream waves. J. Geophys. Res. 92 (A12), 13597-13612.

Schubert, G., Anderson, J.D., Spohn, T., McKinnon, W.B., 2004. Interior composition, structure and dynamics of the Galilean satellites. In: Bagenal, F., Dowling, T.,
McKinnon, W.B. (Eds.), Jupiter: The Planet, Satellites, and Magnetosphere. Cambridge Univ. Press, UK, pp. 281-306.

Schumann, W.O., 1952. Über die strahlungslosen Eigenschwingungen einer leitenden Kugel, die von einer Luftschicht und einer Ionosphärenhülle umgeben ist, (On the free oscillations of a conducting sphere which is surrounded by an air layer and an ionosphere shell). Zeitschrift für Naturforschung 7a, 149-154.

Sentman, D.D., 1990a. Electrical conductivity of Jupiter's shallow interior and the formation of a resonant planetary-ionospheric cavity. Icarus 88, 73-86.

Sentman, D.D., 1990b. Approximate Schumann resonance parameters for a twoscale height ionosphere. J. Atmos. Terr. Phys. 52, 35-46.

Sentman, D.D., 1995. Schumann resonances. In: Volland, H. (Ed.), Handbook of Atmospheric Electrodynamics, Atmospheric Electricity, vol. 1. CRC Press Inc., London, Tokyo, pp. 267-298.

Schröder, S.E., 2007. Investigating the surface of Titan with the Descent Imager/ Spectral Radiometer onboard Huygens. Ph.D. Thesis, Göttingen University, Germany.

Simões, F. et al., 2007. A new numerical model for the simulation of ELF wave propagation and the computation of eigenmodes in the atmosphere of Titan: Did Huygens observe any Schumann Resonance? Planet. Space Sci. 55, 19781989.

Sittler, E.C. et al., 2009. Energy deposition processes in Titan's upper atmosphere and its induced magnetosphere. In: Brown, R.H., Lebreton, J.P., Waite, J.H. (Eds.), Titan from Cassini-Huygens. Springer, Dordrecht, Heidelberg, London, NewYork, p. 535

Smith, B.A. et al., 1981. Encounter with Saturn: Voyager 1 imaging science results. Science 212, 163-191.

Sotin, C., Mitri, G., Rappaport, N., Schubert, G., Stevenson, D., 2009. Chapter 4: Titan's Interior Structure. In: Brown, R.H., Lebreton, J.-P., Waite, H. (Eds.), Titan. Springer-Verlag, pp. 61-73.

Stiles, B.W. et al., 2008. Determining Titan's spin state from Cassini/ RADAR images. Astron. J. 135, 1669-1680.

Stiles, B.W. et al., 2010. Erratum: “Determining Titan's spin state from Cassini RADAR images" (Astron. J. 2008;135:1669). Astron. J. 139, 311.

Tobie, G., Grasset, O., Lunine, J.I., Mocquet, A., Sotin, C., 2005. Titan's internal structure inferred from a coupled thermal-orbital model. Icarus 175, 496-502.

Tokano, T., Neubauer, F.M., 2005. Wind-induced seasonal angular momentum exchange at Titan's surface and its influence on Titan's length-of-day. Geophys. Res. Lett. 32, L24203. doi:10.1029/2005GL024456.

Tokano, T., Van Hoolst, T., Karatekin, Ö., 2011. Polar motion of Titan forced by the atmosphere. J. Geophys. Res. 116, E05002. doi:10.1029/2010JE003758, 2011.

Tomasko, M.G., West, R.A., 2009. Aerosols in Titan's atmosphere. In: Brown, R.H et al. (Eds.), Titan from Cassini-Huygens. Springer, Dortrecht, London, NewYork, pp. 97-321, ISBN:978-1-4020-9214-5.

Wait, J.R., 1962. Electromagnetic Waves in Stratified Media. Pergamon Press, Oxford, London, New-York, Paris, 372pp.

Walterscheid, R.L., Schubert, G., 2006. A tidal explanation for the Titan haze layers. Icarus $183,471-478$.

Wye, L.C., Zebker, H.A., Ostro, S.J., West, R.D., Gim, Y., Lorenz, R.D., the Cassini RADAR Team, 2007. Electrical properties of Titan's surface from Cassini Radar scatterometer measurements. Icarus 188, 367-385.

Zarnecki, J.C. et al., 2005. A soft solid surface on Titan as revealed by the Huygens Surface Package. Nature 438, 792-795. 